\title{
Macrophage-derived oncostatin M contributes to human and mouse neurogenic heterotopic ossifications
}

Frédéric Torossian, ${ }^{1}$ Bernadette Guerton, ${ }^{1}$ Adrienne Anginot, ${ }^{1}$ Kylie A. Alexander, ${ }^{2}$ Christophe Desterke, ${ }^{3}$ Sabrina Soave, ${ }^{1}$ Hsu-Wen Tseng, ${ }^{2}$ Nassim Arouche, ${ }^{1}$ Laetitia Boutin, ${ }^{1}$ Irina Kulina, ${ }^{2}$ Marjorie Salga, ${ }^{2,4}$ Beulah Jose, ${ }^{2}$ Allison R. Pettit, ${ }^{2}$ Denis Clay, ${ }^{3}$ Nathalie Rochet, ${ }^{5}$ Erica Vlachos, ${ }^{6}$ Guillaume Genet, ${ }^{6}$ Charlotte Debaud, ${ }^{4,6}$ Philippe Denormandie, ${ }^{6}$ François Genet, ${ }^{4,6}$ Natalie A. Sims, ${ }^{7}$ Sébastien Banzet, ${ }^{1,8}$ Jean-Pierre Levesque, ${ }^{2}$ Jean-Jacques Lataillade, ${ }^{1,8}$ and Marie-Caroline Le Bousse-Kerdilès ${ }^{1}$

IInserm UMR-S-MD1197, Paris 11 University, Paul Brousse Hospital, Villejuif, France. ${ }^{2}$ Mater Research Institute, The University of Queensland, Translational Research Institute, Woolloongabba, Queensland, Australia. ${ }^{3}$ UMS33, Paris 11 University, Paul Brousse Hospital, Villejuif, France. ${ }^{4}$ Université de Versailles Saint-Quentin-en-Yvelines, Evolution of neuromuscular diseases: innovative concepts and practices, Inserm U1179, Montigny le Bretonneux, France. ${ }^{5}$ Université Côte d'Azur, CNRS, Inserm, Institut de Biologie Valrose, Nice, France. ${ }^{6}$ Service de Médecine Physique et de Réadaptation, Paris 12 University, Garches, France. ${ }^{75 t}$. Vincent's Institute of Medical Research and Department of Medicine, St. Vincent's Hospital, The University of Melbourne, Fitzroy, Victoria, Australia. ${ }^{8}$ Centre de Transfusion Sanguine des Armées, L'Institut de Recherche Biomédicale des Armées, Clamart, France.

Neurogenic heterotopic ossification (NHO) is the formation of ectopic bone generally in muscles surrounding joints following spinal cord or brain injury. We investigated the mechanisms of $\mathrm{NHO}$ formation in 64 patients and a mouse model of spinal cord injury-induced NHO. We show that marrow from human NHOs contains hematopoietic stem cell (HSC) niches, in which mesenchymal stromal cells (MSCs) and endothelial cells provide an environment supporting HSC maintenance, proliferation, and differentiation. The transcriptomic signature of MSCs from NHOs shows a neuronal imprinting associated with a molecular network required for HSC support. We demonstrate that oncostatin M (OSM) produced by activated macrophages promotes osteoblastic differentiation and mineralization of human muscle-derived stromal cells surrounding NHOs. The key role of OSM was confirmed using an experimental model of $\mathrm{NHO}$ in mice defective for the OSM receptor (OSMR). Our results provide strong evidence that macrophages contribute to NHO formation through the osteogenic action of OSM on muscle cells within an inflammatory context and suggest that OSM/OSMR could be a suitable therapeutic target. Altogether, the evidence of HSCs in ectopic bones growing at the expense of soft tissue in spinal cord/brain-injured patients indicates that inflammation and muscle contribute to HSC regulation by the brain-bone-blood triad.

Authorship note: J.P. Levesque, J.J. Lataillade, and M.C. Le BousseKerdilès are co-senior authors.

Conflict of interest: The authors have declared that no conflict of interest exists.

Submitted: June 30, 2017 Accepted: September 26, 2017 Published: November 2, 2017

Reference information: JCI Insight. 2017;2(21):e96034. https://doi.org/10.1172/jci. insight. 96034.

\section{Introduction}

Heterotopic ossifications (HOs) are characterized by the formation of ectopic bone in soft tissues surrounding joints: particularly the hip, knee, elbow, or shoulder. Genetic hereditary forms of HOs, such as fibrodysplasia ossificans progressiva (FOP) (1-4), are extremely rare (1:2,000,000 prevalence). However, HOs can have a much higher incidence following severe trauma, such as traumatic brain or spinal cord injuries (SCIs), stroke and cerebral anoxia (neurogenic $\mathrm{HO}$ [NHO]) (5), fractures, or extended severe burns.

Patients with HOs suffer from pain and highly reduced range of motion from affected joints, leading to complete ankyloses on affected joints. Currently, the only effective treatment for HOs is surgical resection, but this is only temporarily effective, since HOs can reoccur, even 15 years after resection (6). Furthermore, complications associated with resection occur when ossifications entrap large blood vessels and nerves. A better understanding of $\mathrm{HO}$ etiology is required to develop more lasting treatments or a cure (7).

Hematopoietic marrow-like tissue has been observed in the center of HOs on histological sections, as described in two case reports $(8,9)$. Recently, the presence of clonogenic hematopoietic progenitors 
associated with histologically defined stromal cells has been reported in HOs from severe combat-injured orthopedic patients (10). Likewise, in a mouse model of SCI-induced NHO, which we recently developed, hematopoietic marrow-like tissue was observed in the center of NHOs (11). However, we believe that until now, the presence of a hematopoietic stem cell (HSC) niche, defined by functional HSCs, mesenchymal stromal cells (MSCs), and endothelial cells (12), has never been demonstrated in NHO marrow.

Osteogenic precursor cells are necessary for ectopic osteogenesis in soft tissues (13). Cells from several origins have been proposed to be implicated in the formation of ectopic bones in traumatic or hereditary HOs (14). First, circulating osteogenic precursors have been reported to contribute to HO formation in patients with FOP (15), confirming a previous report in a mouse model in which ectopic ossification was induced by collagen implants containing exogenous recombinant BMP-2 (16). Second, resident muscle cells with osteogenic differentiation capacity have also been proposed. Indeed, Tie ${ }^{+} \mathrm{P}-$ $\mathrm{DGFR} \alpha^{+} \mathrm{Sca}-1^{+}$progenitors that reside in the murine skeletal muscle interstitium generated ectopic bone in a similar BMP-2-dependent model of $\mathrm{HO}(17)$. Osteogenic differentiation capacity of PDGFR $\alpha^{+}$ cells isolated from healthy human skeletal muscles was confirmed in vivo when they were implanted subcutaneously on hydroxyapatite scaffolds (18). A third possible source of precursors is a cell population with a $\mathrm{CD} 73^{+} \mathrm{CD} 105^{+} \mathrm{CD} 90^{+} \mathrm{MSC}$ phenotype, capable of in vitro adipogenic, osteogenic, and chondrogenic (AOC) differentiation, which was isolated from combat-injured muscle from wounded military personnel; however, there is no functional in vivo study to prove that these cells were at the origin of combat injury-induced HOs (19). Moreover, the injured muscles that subsequently developed $\mathrm{HO}$ also demonstrated increased numbers of osteogenic progenitors (20). In contrast, Downey et al. (21) demonstrated that in healthy muscles, $\mathrm{CD} 73^{+} \mathrm{CD} 105^{+} \mathrm{CD} 90^{-}$cells rather than $\mathrm{CD} 73^{+} \mathrm{CD} 105^{+} \mathrm{CD} 90^{+}$ cells displayed robust AOC differentiation. Therefore, to date, the nature of osteogenic precursor cells forming HOs remains to be defined, especially in NHOs.

Several growth factors have been suggested to be involved in $\mathrm{HO}$ development. Among them are BMPs, a group of signaling molecules in the TGF- $\beta$ superfamily that regulate bone organogenesis (22). Their participation in HO genesis was implied when intramuscular injection of BMP-9 in damaged skeletal muscles in mice induced ectopic bone formation (23). The description of animal models of HOs in which BMPs are required for induction of ectopic bone $(24,25)$ and the presence of a mutation in the ACVR1 gene that encodes the BMP type I receptor in FOP (1) strengthen the evidence for BMPs in the pathology of hereditary HOs such as FOP.

Apart from BMPs, others factors, including inflammatory cytokines and chemokines, could also participate in both hereditary and trauma-induced $\mathrm{HO}$ development $(26,27)$. We have recently described the triggering effect of macrophage-mediated inflammation in muscles in a SCI-induced NHO mouse model (11). Among cytokines produced by macrophages in an inflammatory context, oncostatin M (OSM) was upregulated after SCI in mice and it exhibited a neuroprotective effect against SCI or ischemic stroke (28, 29). OSM belongs to the IL-6 cytokine family, which includes IL-11, leukemia inhibitory factor (LIF), cardiotrophin-1, ciliary neurotrophic factor, and neuropoietin (30). OSM binds 2 receptor complexes: type I, composed of gp130 and LIF receptor subunits (31), and type II, composed of gp130 and OSM receptor (OSMR) subunits (32). OSM is secreted by hematopoietic cells, such as monocytes, macrophages, dendritic cells, T cells, or neutrophils (33-35), but also by osteoblasts, osteocytes, and microglia (36-38). Its secretion is increased upon stimulation by LPS, GM-CSF, or prostaglandin E2 $(34,35,37,39)$. OSM is able to stimulate the osteogenic differentiation of MSCs isolated from bone marrow (BM-MSCs) as well as from adipose tissue $(39,40)$ and to regulate osteoblast and osteoclast activities $(33,38)$. Furthermore, release of OSM by activated macrophages promotes differentiation of osteoblasts in vitro $(41,42)$. By playing multiple critical roles in the hematopoietic microenvironment, OSM has also been reported to participate in HSC regulation in the bone marrow (BM) $(43,44)$.

From a cohort of 64 patients with brain injury or SCI, we show in this study that NHOs contain functional HSC niches in which a mesenchymal and endothelial environment allows HSC maintenance, proliferation, and differentiation. Moreover, we demonstrate that activated macrophages contribute to bone formation through the osteogenic action of OSM on muscle cells within an inflammatory context. Finally, we show in a mouse model of SCI-induced NHO that we have recently developed (11) that SCI increases OSM expression in injured muscle and promotes the osteogenic potential of sorted muscle progenitor cells in vitro and that deletion of the Osmr gene reduces $\mathrm{NHO}$ development in vivo. 


\section{Results}

Human NHOs contain functional HSCs. In our study, 70 NHO samples from 64 patients were surgically resected and 66 of them were sectioned. Hematopoietic sites associated with osteoblasts/osteocytes, chondrocytes, and adipocytes were observed on sections of 39 of these 66 NHO biopsies (59\%) after H\&E staining (Figure 1A). Presence of hematopoietic cells was confirmed by FACS analysis after labeling mononuclear cells isolated from NHOs (Figure 1B). Presence of leukocytes coexpressing CD45 and CD15, CD19, or CD11/CD14 antigens indicated the existence of neutrophils, B lymphocytes, and monocytes/macrophages, respectively, in NHOs (Figure $1 \mathrm{~B}$ ). $\mathrm{CD}^{+} \mathrm{CD}^{+}$and $\mathrm{CD} 3^{+} \mathrm{CD} 8^{+} \mathrm{T}$ lymphocytes were also present. Detection of $\mathrm{CD} 45^{+} \mathrm{CD} 34^{+}$cells suggested the presence of hematopoietic stem/progenitor cells (HSPCs) (Figure 1B). The percentage of $\mathrm{CD} 34^{+}$cells in the $\mathrm{CD} 45^{+}$mononuclear cell fraction was heterogeneous but slightly lower in NHOs $(2.47 \% \pm 0.52 \%, n=14)$ than in the BM $(4.43 \% \pm 1.22 \%, n=3)$ from healthy donors (HD). However, the CD34 $4^{+}$HSPC frequency was about 20-fold higher in NHOs than in the blood from $\operatorname{HDs}(2.47 \% \pm 0.52 \%, n=14$, compared with $0.110 \% \pm 0.01 \%, n=3$, respectively) (Figure $1 \mathrm{C})$. To analyze the presence of hematopoietic progenitor cells in $\mathrm{CD}_{3} 4^{+}$cells purified from NHOs, we performed clonogenic assays. Multipotent, erythroid, granulocytic, and macrophage progenitors could be detected in NHOs (Figure 1D) as well as progenitors of megakaryocytes (data not shown). The percentage of each colony type was similar in NHOs and in BM from HDs.

We further investigated the presence of side population (SP) cells, which are enriched in quiescent HSCs and characterized by their high capacity to efflux Hoechst33342 dye (45). As in BM, SP cells with a low Hoechst 33342 fluorescence intensity could be detected in the Lin ${ }^{-}$cells isolated from NHOs (Figure 1E). The specificity of the SP population was demonstrated by its selective loss after addition of verapamil, an inhibitor of ABC transporter proteins that are responsible for Hoechst33342 efflux. A small proportion of these SP cells expressed the $\mathrm{CD} 45^{+} \mathrm{CD} 34^{+} \mathrm{CD} 38^{-}$phenotype (Figure 1E) of immature HSPCs.

$\mathrm{CD}_{34}{ }^{+}$cells isolated from human NHOs were intravenously injected into nonobese diabetic severe combined immune-deficient $\mathrm{Il}_{2 \mathrm{rg}^{-1-}}$ (NSG) mice. Eight weeks after transplantation, the engraftment capability of $\mathrm{CD} 34^{+}$cells from NHOs in NSG mice was demonstrated with the presence of human $\mathrm{CD} 45^{+} \mathrm{CD} 19^{+}$ lymphoid and $\mathrm{CD} 45^{+} \mathrm{CD} 15^{+}$or $\mathrm{CD} 45^{+} \mathrm{CD} 11 \mathrm{~b}^{+}$myeloid cells as well as $\mathrm{CD} 45^{+} \mathrm{CD} 34^{+}$cells in the mouse BM (Figure $1 \mathrm{~F}$ and Supplemental Table 1). The evidence of a small population of human CD $45^{+} \mathrm{CD} 19^{+}$ cells after a secondary graft in NSG mice (Supplemental Figure 1; supplemental material available online with this article; https://doi.org/10.1172/jci.insight.96034DS1) definitively confirmed the presence of human HSCs in human NHOs.

Human NHOs are HSC niches with a stroma supporting in vitro and in vivo hematopoiesis. We next studied the endothelial and mesenchymal stromal microenvironment of human NHOs and analyzed whether, as in BM from HDs, it could support functional hematopoiesis. We first investigated whether functional endothelial progenitors were detected and isolated from NHOs. Endothelial CD31+ cells were immunomagnetically separated and then sorted as $\mathrm{CD} 31^{+} \mathrm{CD} 144^{+} \mathrm{CD} 34^{+} \mathrm{CD} 45^{-}$cells (Figure 2A). Sorted cells were able to form colonies and grow in culture as a cobblestone monolayer, a typical morphology of endothelial cells (Figure 2B). After 1 month of culture, these cells still exhibited the $C D 31^{+} \mathrm{CD} 144^{+} \mathrm{CD} 34^{+} \mathrm{CD} 45^{-}$endothelial cell phenotype devoid of $\mathrm{CD}^{+} 5^{+}$leukocytes (Figure 2C). CD34 antigen expression was heterogeneous on these cells and was correlated with that of the CD31 antigen. As confirmation of their endothelial phenotype, sorted cells cultured in Matrigel were able to form vascular networks (Figure 2D). Moreover, expression of adhesion molecules VCAM-1 and ICAM-1 was increased after stimulation with proinflammatory cytokine TNF- $\alpha$ (Figure 2E), demonstrating the functional capabilities of endothelial cells isolated from NHOs.

We also isolated adherent stromal cells from NHOs, cultured them for 2 passages, and tested the expression of MSC markers by flow cytometry. As expected, they exhibited the classical mesenchymal CD45- ${ }^{-}$D $34-\mathrm{CD} 73^{+} \mathrm{CD} 90^{+} \mathrm{CD} 105^{+}$phenotype (Figure $2 \mathrm{~F}$ ). These cells were then induced to differentiate into the three AOC mesenchymal lineages using specific media, as shown by staining, confirming that adherent cells from NHOs were MSCs (NHO-MSCs; Figure 2G). The osteogenic potential of NHO-MSCs was further demonstrated by Western blot analysis showing expression of Runx2, a transcription factor required for osteoblast differentiation, and bone sialoprotein II (BSPII), a component of mineralized tissues (Figure $2 \mathrm{H}$ ).

We then compared the transcriptomic profile of NHO-MSCs and normal BM-MSCs (GEO GSE94683). Gene set enrichment analysis performed according to a transcriptomic profile compatible 
A

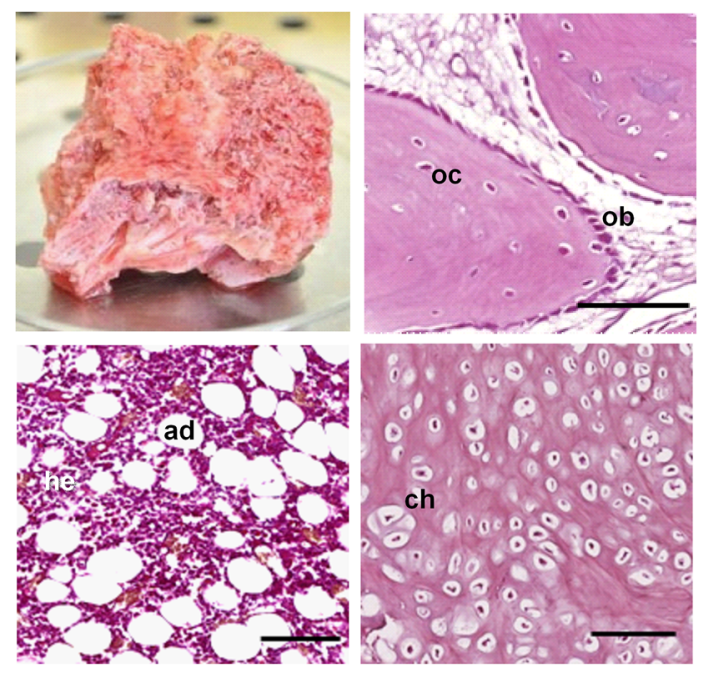

B

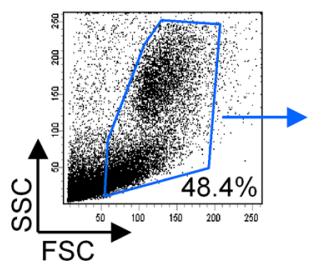

C

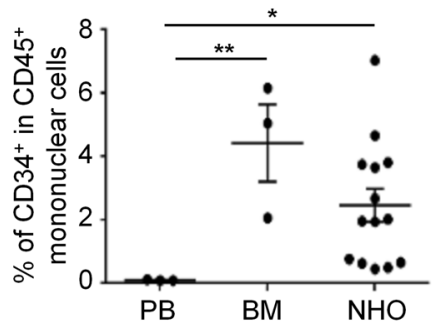

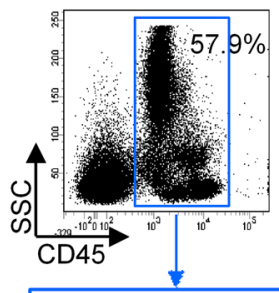

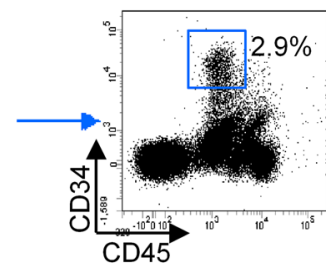

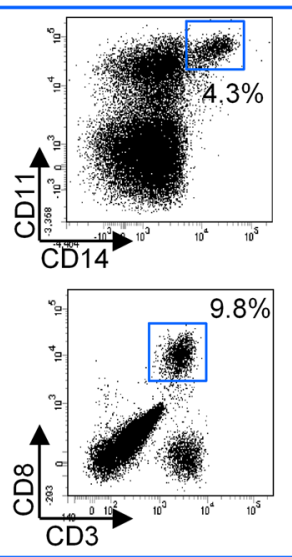

D
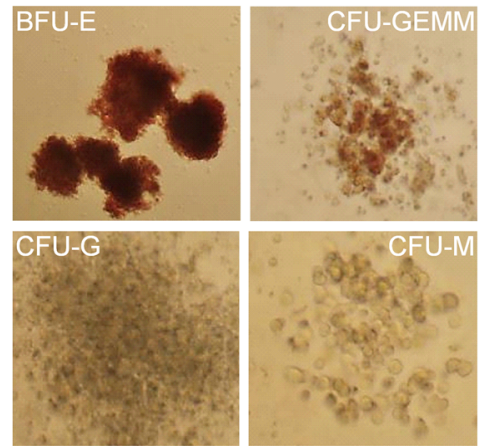

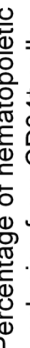

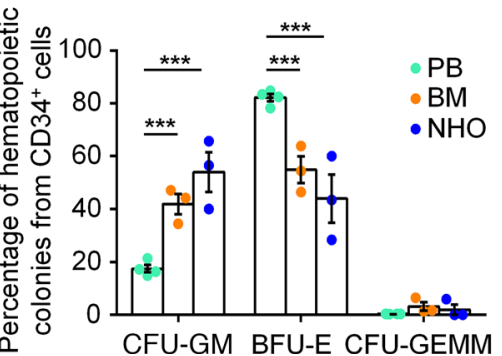

$\mathbf{E}$
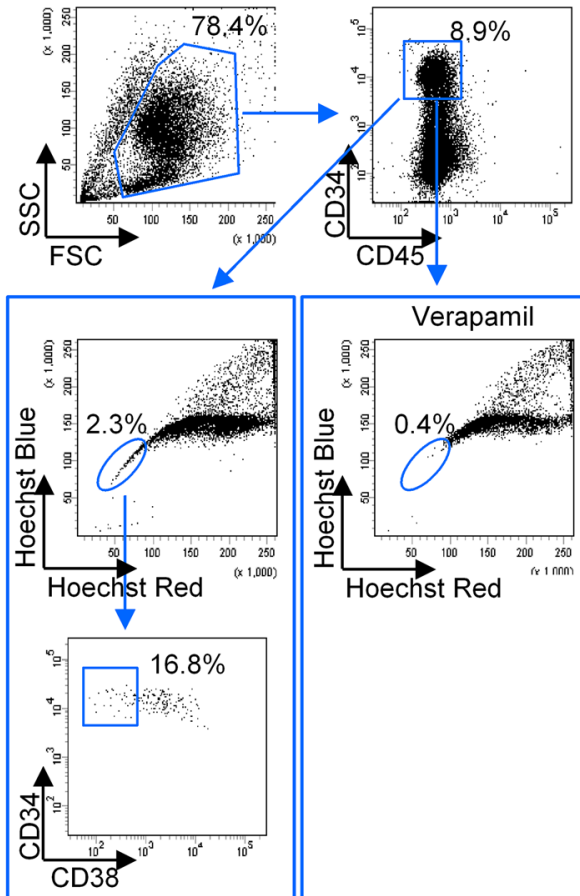

$\mathbf{F}$
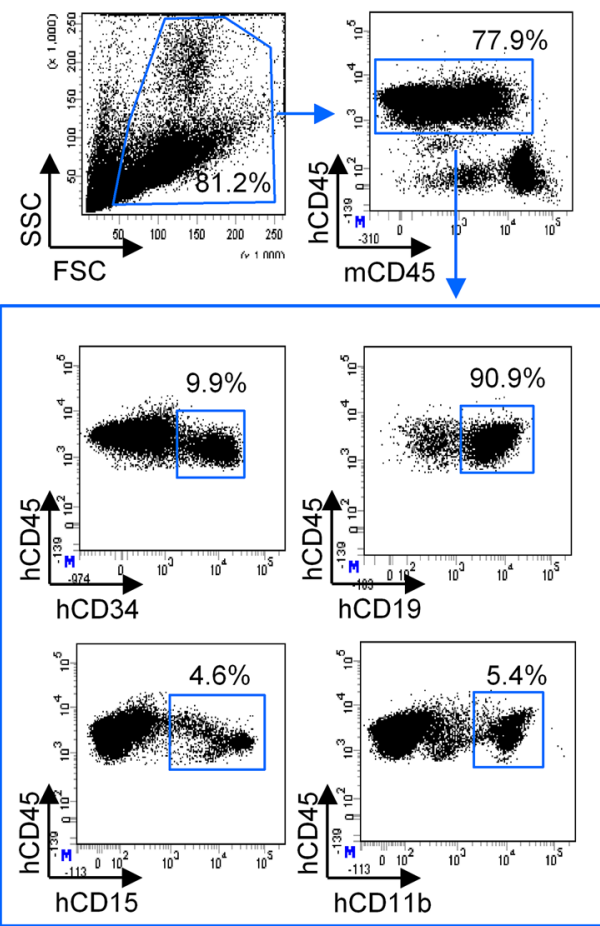

Figure 1. Human NHOs contain functional hematopoietic stem cells. (A) Fragment of human neurogenic heterotopic ossification (NHO) with hematopoietic activity resected from a patient with stroke. Osteoblasts (ob), osteocytes (oc), hematopoietic cells (he), adipocytes (ad), and chondrocytes (ch) on sections of human NHO biopsies stained with H\&E. Scale bar: $100 \mu \mathrm{m}$. (B) Flow cytometry characterization on human mononuclear leukocytes from one hematopoietic NHO representative of the 5-14 analyzed. (C) Percentage of CD34+ cells within the CD45 mononuclear leukocyte fraction from peripheral blood (PB) and bone marrow (BM) from healthy donors and from NHOs. Each dot represents a different donor/patient. Bars represent mean \pm SEM ( $n=3-14)$. Kruskal-Wallis test followed by Dunn's post-hoc tests were used for the statistical analysis ( ${ }^{*} P \leq 0.05$, $\left.{ }^{*} P \leq 0.01\right)$. (D) CD34 ${ }^{+}$cells from PB, BM, and $\mathrm{NHOs}$ were isolated using immunomagnetic cell separation and plated in colony assay with human cytokines. Images show representative colonies from erythroid (BFU-E), granulocyte/erythroid/macrophage/megakaryocyte (CFU-GEMM), granulocytic (CFU-G), and macrophage (CFU-M) progenitors. Data show relative frequencies of granulocyte/macrophage (CFU-CM), BFU-E, and CFU-GEMM progenitors after 14 days of culture ( $n=3-4$ patients/donors per tissue). Results are expressed as mean \pm SEM. Two-way ANOVA followed by Tukey's post-hoc tests were used (**P $\leq 0.001)$. (E) NHOs contain side population (SP) CD34+ cells. Lineage-negative cells from NHOs were isolated using immunomagnetic cell separation. SP cells (blue circles) were analyzed by flow cytometer with or without $50 \mu \mathrm{M}$ verapamil for expression CD34 and CD38 markers $(n=3)$. (F) CD34+ cells from human NHOs reconstitute human hematopoiesis in immunodeficient mice. CD34+ cells from human NHOs were transplanted intravenously into NSG mice. After 2 months, BM from NSG mice was analyzed by flow cytometry for expression human CD45, CD34, B lymphoid CD19, and myeloid CD11b and CD15 markers $(n=6)$. 
A

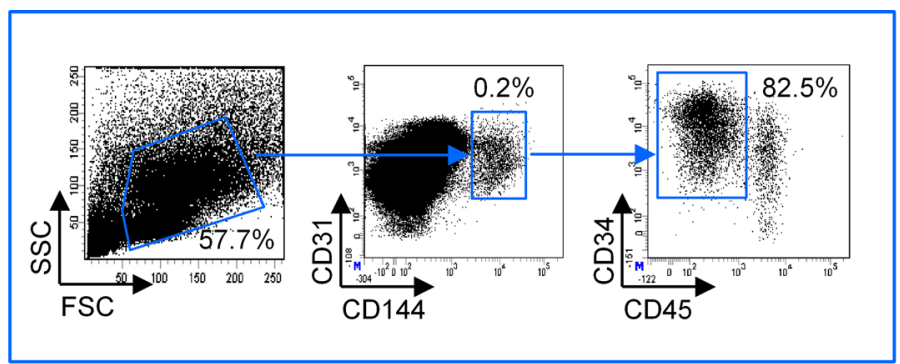

B

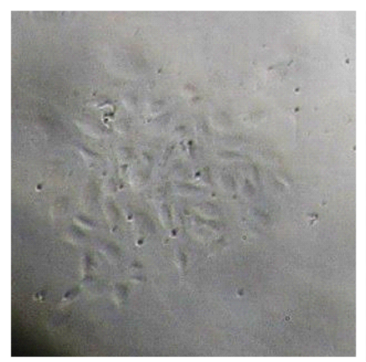

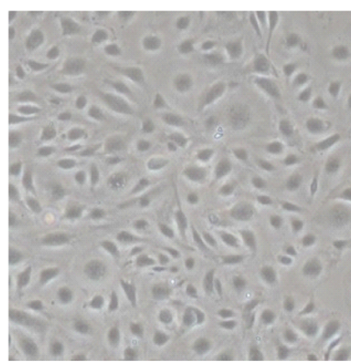

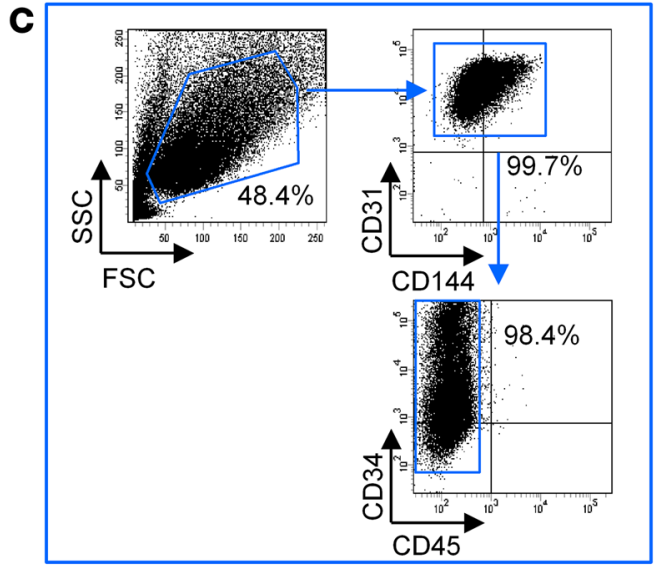

D

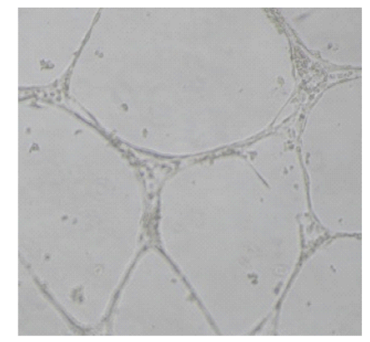

E

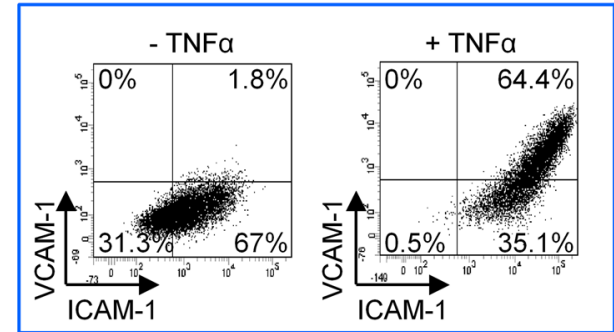

$\mathbf{F}$

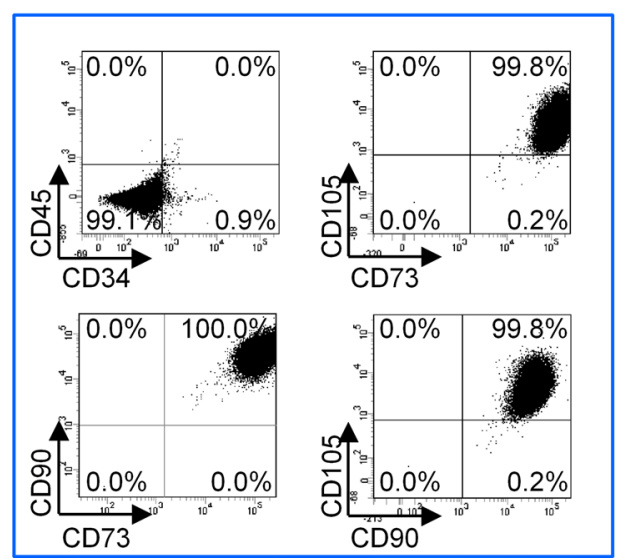

G

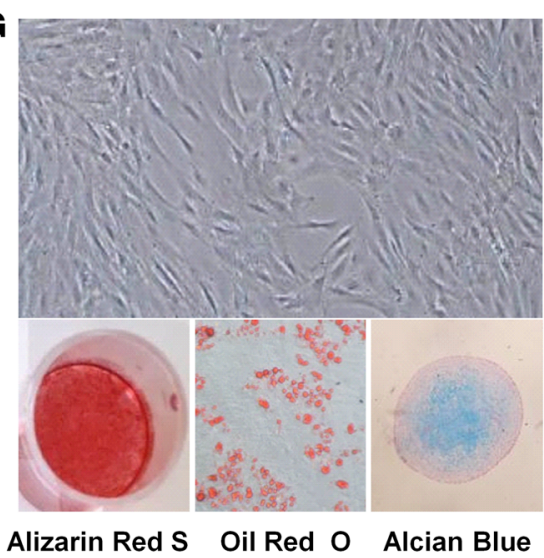

H

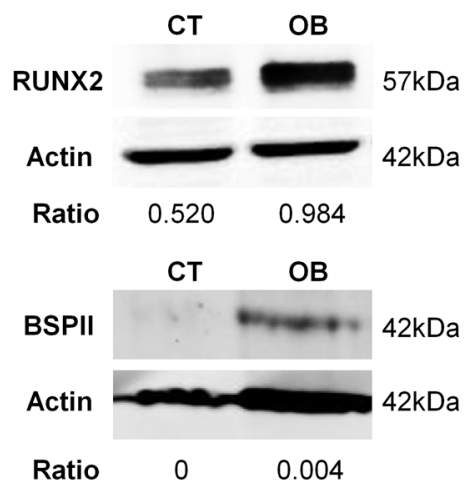

Figure 2. Human NHOs contain endothelial and mesenchymal niche forming cells. (A) Sorting of human neurogenic heterotopic ossification (NHO) marrow endothelial cells. After CD31+ immunomagnetic cell enrichment $(n=2)$, endothelial cells were sorted using the CD $31^{+}$CD $144^{+}$CD $34^{+} C D 45^{-}$phenotype. (B) Sorted CD31+CD144+CD34+CD45- NHO cells formed colonies and grew in a cobblestone monolayer characteristic of endothelial cell morphology. (C) After a month in culture, the phenotype of human NHO endothelial cells was verified by a flow cytometer after labeling for CD31, CD144, CD34, and CD45. (D) Human NHO endothelial cells were able to form a tube network when cultured in Matrigel. (E) VCAM-1 and ICAM-1 expression is increased on human NHO endothelial cells after simulation with TNF- $\alpha(10 \mathrm{ng} / \mathrm{ml})$. (F) Adherent cells isolated from human NHO were analyzed for classical mesenchymal markers by flow cytometry $(n=4)$. (G) Adherent cells were isolated in culture from human NHO and induced to differentiate into the 3 classical mesenchymal lineages using specific inductive media $(n=4)$. Differentiation into osteoblasts, adipocytes, and chondrocytes was evaluated by Alizarin Red S, Oil Red O, and Alcian blue staining, respectively. (H) Western blot showing Runx2 and BSPII protein expression in NHO-MSC cell lysates with (OB) or without (control [CT]) osteoblastic differentiation medium (day 3 and day 21, respectively). Ratios correspond to RUNX2/actin or BSPII/actin. Original magnification, $\times 4$ (B, left); $\times 10$ (B, right; D; and $\mathbf{C})$.

with hematopoietic niche support (46) segregated NHO from BM samples. As shown in Figure 3, A and $\mathrm{B}, 45$ of the $289 \mathrm{BM}$ hematopoietic niche-supportive gene signatures were found overexpressed in NHO samples as compared with BM samples (Figure 3, A and B, and Supplemental Table 2). Unsupervised principal component analysis performed with BM niche-related genes that were enriched in NHO significantly discriminated NHO from BM (Figure 3C). WikiPathway database enrichment highlighted cell functionalities implicated in neuronal and glial biology, the stem cell pathway, and pathways involved in 
bone and cartilage metabolism, including osteopontin signaling, Wnt signaling, endochondral ossification, and matrix metalloproteinases (Figure 3D). Conjointly, analysis of curated literature demonstrated that genes related to the hematopoietic niche segregated two distinct clusters for NHO-MSCs and BM-MSCs by unsupervised classification (Euclidean distances, Figure 4A and Supplemental Table 3). This clustering was also found significant by unsupervised principal component analysis (Figure 4B). Functional enrichment analysis (WikiPathway database) performed with BM niche-related genes highlighted enrichment for genes known to be modulated after SCI (Figure 4C) in NHO-MSCs versus BM-MSCs, such as RAC1, RHOA, CDKN1B, and GDNF (Figure 4D) as well as genes essential to support hematopoiesis and HSPC development, especially KITLG (Figure 4A). The fact that KITLG is expressed at higher levels in NHOMSCs is highly suggestive of the formation of ectopic hematopoietic niches within NHO marrows.

We further analyzed the in vitro and in vivo capability of NHO-MSCs to support functional hematopoiesis. We first demonstrated their in vitro potential to support long-term culture-initiating cell cultures (47) and their capability to maintain primitive hematopoietic progenitor cells, as a higher number of hematopoietic colonies was detected after 5 weeks of coculture compared with cultures without NHOMSC support (Figure 3E). NHO-MSCs were also capable of inducing the SP phenotype of Lin- cells from peripheral blood (Figure 3F), as recently described with BM-MSCs (48). SP cells were CD $45^{+} \mathrm{CD} 34^{+}$, confirming their hematopoietic origin. Finally, we tested whether NHO-MSCs were able to support in vivo hematopoiesis. For this purpose, NHO-MSCs were seeded in hydroxyapatite/calcium phosphate scaffolds and implanted subcutaneously into the backs of nude mice. Ten weeks after implantation, bone tissue containing osteocytes and with hematopoietic foci was observed in implants, indicating that human NHO-MSCs support both bone formation and hematopoiesis in immunodeficient mice (Figure 3G). In contrast, no bone and hematopoietic tissue formation was observed in scaffolds implanted without NHOMSCs (Supplemental Figure 2). Expression of specific human lamin A/C (18) in osteocytes within the heterotopic bone demonstrated that some of them were of human origin (Figure $3 \mathrm{H}$ ), whereas, as excepted, hematopoiesis was of murine origin, similar to previous observations in mice implanted with scaffolds seeded with cultured human BM-MSCs (49). As control, murine BM sections did not exhibit any positive lamin A/C staining (Supplemental Figure 3). Therefore, human NHOs contain functional MSCs capable of forming bone tissue and supporting in vitro and in vivo hematopoiesis.

Altogether, our data demonstrate that the NHO marrow fits with the definition of a stem cell niche in which a functional mesenchymal and endothelial environment supports HSC maintenance, proliferation, and differentiation.

Muscle-derived stromal cells surrounding human NHOs exhibit osteogenic differentiation potential in response to inflammatory stimuli. HOs are reported to develop from degenerating muscle fibers within an inflammatory context (50). Therefore, we investigated whether cells with osteogenic differentiation potential could be isolated from the surrounding muscles in which NHOs grow. After explant culture of these muscles, adherent cells from muscles surrounding NHOs were expanded by 2 passages and analyzed for phenotypic and functional characterization. Muscle-derived stromal cells (NHO-MDSCs) displayed classical MSC morphology and a $\mathrm{CD} 73^{+} \mathrm{CD} 90^{+} \mathrm{CD} 105^{+}$phenotypic profile (Figure 5, A and B). As expected, these cells were CD45 but heterogeneous in CD34 expression. In contrast to BM-MSCs, which were uniformly CD34-, a proportion of $\mathrm{CD}_{3} 4^{+}$cells $(1.3 \%-17.1 \%)$ was detected in cultures of NHO-MDSCs, suggesting the presence of quiescent satellite cells (SCs) (51) within this NHO-MDSC adherent population. These NHO-MDSCs were able to differentiate into osteoblasts and adipocytes and produce cartilage glycosaminoglycan matrix when cultured in specific inductive media (Figure 5A).

We further investigated the role of inflammation in the osteogenic potential of muscle cells surrounding NHOs. We tested the response of NHO-MDSCs to an inflammatory microenvironment by adding bacterial LPSs or human TNF- $\alpha$ to these cultures. NHO-MDSCs demonstrated osteogenic differentiation potential when induced by osteogenic medium (OB) as compared with noninduced cells (CT), as shown by the presence of mineralized calcium deposit (Figure 5C). Here again, osteoblast differentiation was further documented by Western blot showing the expression of Runx2 and BSPII proteins (Figure 5D). This mineralization was increased when LPS or TNF- $\alpha$ was added to the osteogenic medium (Figure $5 \mathrm{C}$ ). These results suggest that human NHO-MDSCs contain progenitors with osteogenic potential and that inflammatory cytokines play a role in their osteogenic differentiation.

Activated macrophages contribute to the osteogenic differentiation of human NHO-MDSCs. Since we have previously demonstrated in mice that NHO development following SCI is triggered by macrophages 
A

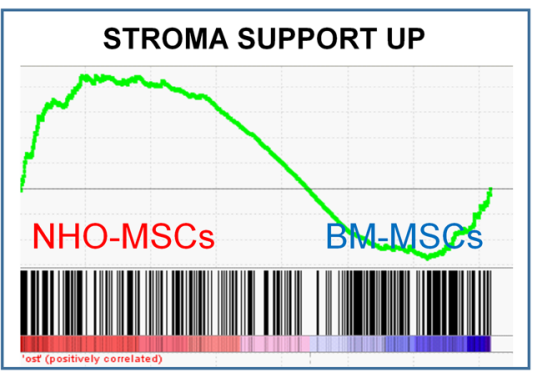

B

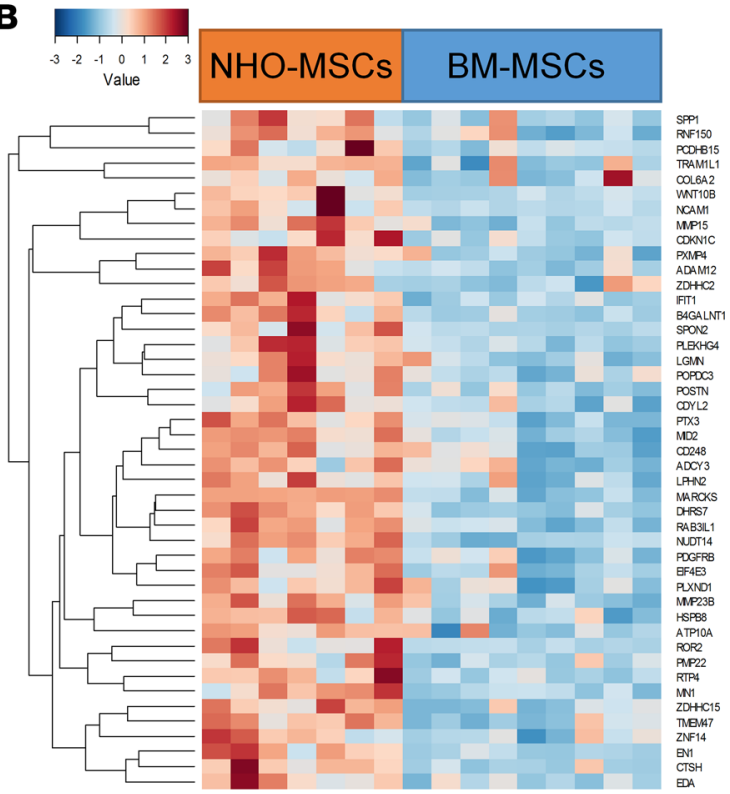

E

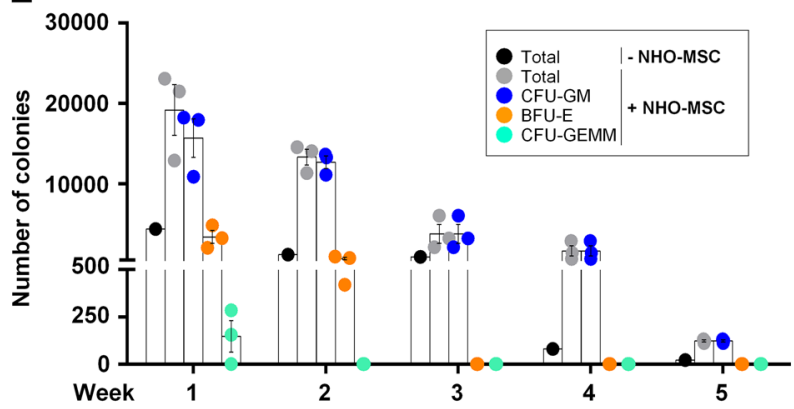

G
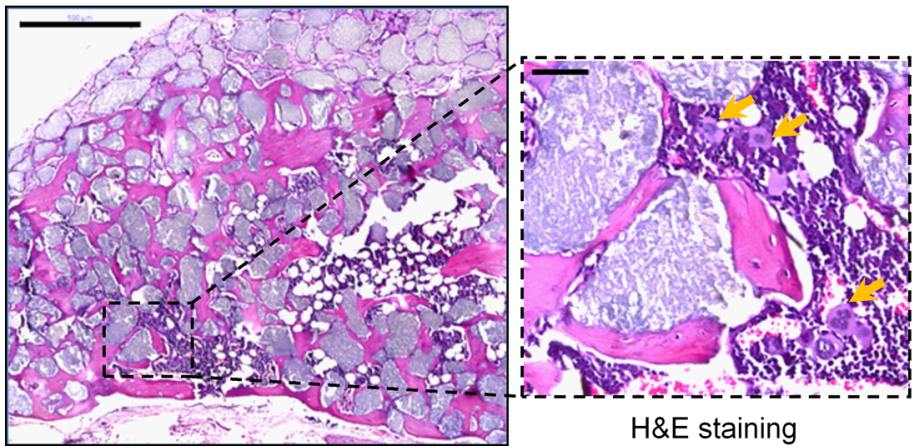

C

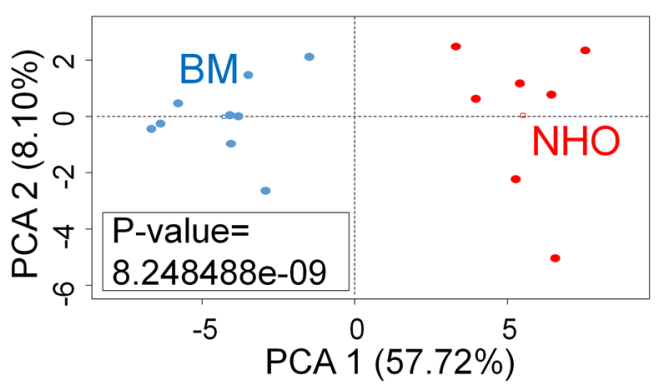

D

Wikipathway 2016

(Negative log(p-value)

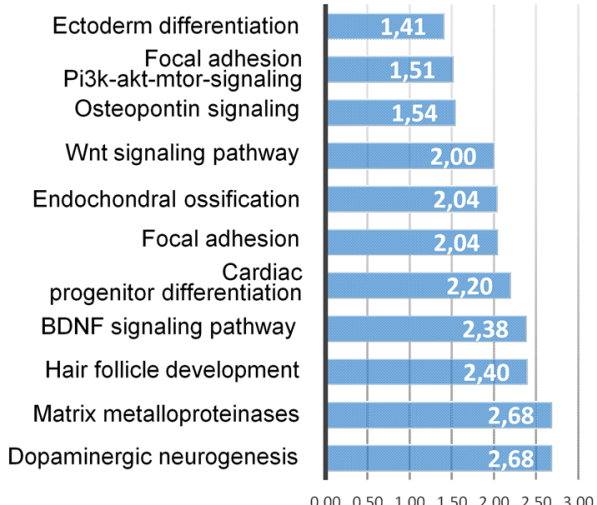

$\mathbf{F}$

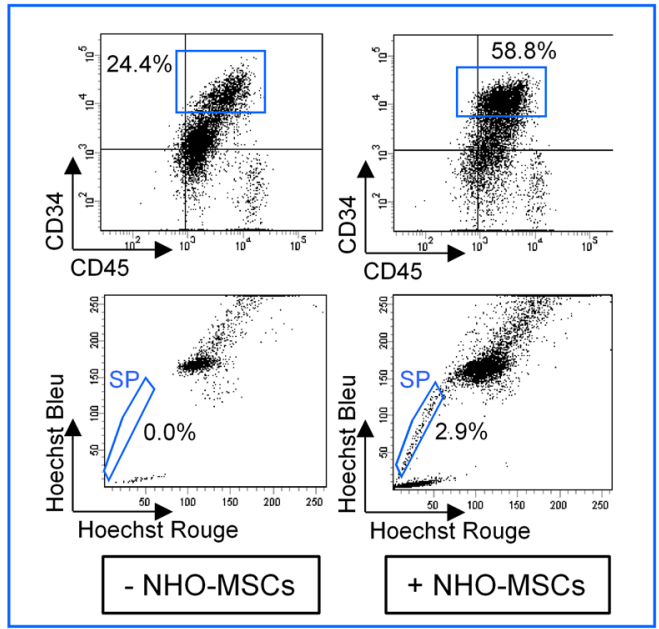

H

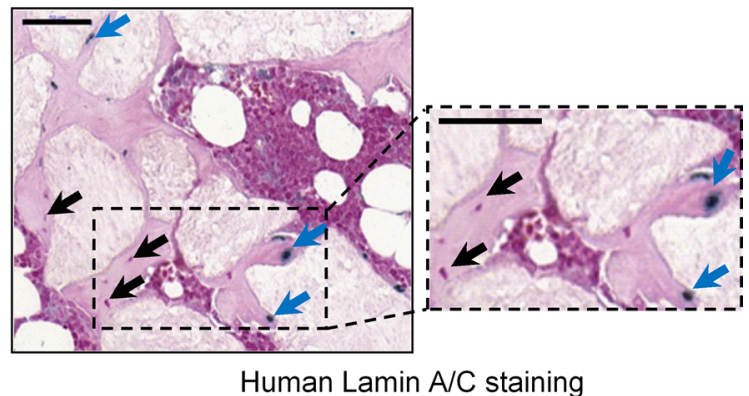

Figure 3. Human NHO-MSCs support in vitro and in vivo hematopoiesis. (A) Transcriptome gene set enrichment analysis performed with the Charbord et al. expression profile (46) was compatible with a hematopoietic niche support and applied to the expression differential between neurogenic heterotopic ossification (NHO; $n=7$ ) and bone marrow (BM; $n=9$ ) mesenchymal stromal cells (MSCs). (B) Heatmap representing hematopoiesis-supporting genes upregulated in NHO-MSCs versus BM-MSCs; each column represents the transcriptome of MSCs from individual patients (NHO-MSCs) or healthy donor controls (BM-MSCs). (C) Unsupervised principal component analysis performed with genes supporting hematopoiesis discriminated NHO-MSCs 
from BM-MSCs ( $P$ value calculated by correlation of the group variable to the first principal axis). (D) Bar plot representing functional enrichment analysis (WikiPathway database) showing hematopoiesis supporting genes overexpressed in NHO-MSCs (bars represent negative logarithm base 10 of the enrichment $P$ value). (E) Number of colonies formed from human BM hematopoietic cells cocultured with NHO-MSCs $(n=3)$ for 5 weeks. Results are expressed as the mean number of colonies for the total number of cells obtained after each weeks of culture $\pm \mathrm{SEM}$. (F) Identification of CD45 ${ }^{+} \mathrm{CD} 34^{+}$side population (SP) cells obtained after a 4-day coculture of lineage-negative cells from peripheral blood with or without human NHO-MSCs. (C) Scaffolds with human NHO-MSCs implanted into nude mice (H\&E). Representative section 10 weeks after implantation in mice $(n \geq 4)$; yellow arrows indicate the presence of megakaryocytes. Scale bar: $500 \mu \mathrm{m}$ (left); $50 \mu \mathrm{m}$ (right). (H) Specific human lamin A/C staining; blue arrows indicate human osteocytes positive for lamin $\mathrm{A} / \mathrm{C}$, and black arrows indicate murine cells. Scale bar: $50 \mu \mathrm{m}$.

infiltrating damaged muscles (11), we studied the role of macrophages in the osteogenic differentiation of human NHO-MDSCs. We first isolated $\mathrm{CD} 34^{+}$cells from human NHOs and induced them to differentiate into NHO-derived macrophages (NHOmacs) using a specific cocktail of cytokines, including human stem cell factor (SCF), thrombopoietin, Flt3-ligand (Flt3L), (IL-3), and colony-stimulating factor 1 (CSF-1) (52). After 2 weeks in culture, NHOmacs contained about 52.38\% $\pm 12.38 \%(n=$ 6) differentiated $\mathrm{CD} 45^{+} \mathrm{CD} 11 \mathrm{~b}^{+} \mathrm{CD} 14^{+}$macrophages that also expressed $\mathrm{M} 2$ macrophage polarization markers CD163 and CD206 (Figure 6A). They were then cocultured with NHO-MDSCs in osteogenic medium with or without LPS $(100 \mathrm{ng} / \mathrm{ml})$ to activate macrophages. Addition of LPS-activated NHOmacs significantly increased mineralization by NHO-MDSCs as compared with osteogenic medium alone (Figure 6B).

$\mathrm{CD} 14^{+}$monocytes/macrophages present in the mononuclear cell fraction from human NHOs (Figure 6C) were further directly isolated using $\mathrm{CD} 14^{+}$immunomagnetic separation and activated with or without LPS $(100 \mathrm{ng} / \mathrm{ml})$ for 3 days. Conditioned medium from LPS-activated CD $14^{+}$monocytes/macrophages $\left(\mathrm{NHOmac} \mathrm{CM}^{+}\right)$or from nonactivated $\mathrm{CD}_{1} 4^{+}$monocytes/macrophages $\left(\mathrm{NHOmac} \mathrm{CM}^{-}\right)$was recovered

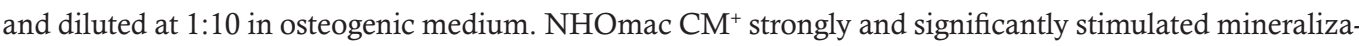
tion of NHO-MDSCs compared with the OB condition (Figure 6D).

Altogether our results indicate that LPS-activated monocytes/macrophages contribute to the mineralization and osteogenic differentiation of NHO-MDSCs through soluble factor production.

Macrophage-derived OSM is involved in human NHO formation. Taking into account the role of activated monocytes/macrophages in the osteogenic differentiation of NHO-MDSCs, we further analyzed whether inflammatory cytokines produced by activated monocytes/macrophages were involved in this osteogenic effect. Among these cytokines, OSM has been reported to promote osteogenesis in MSCs through activation of its receptor $(38,39)$. We therefore analyzed the local production of OSM in NHOs. Figure 7A shows the expression of OSM by several cell types in NHO sections, especially within clusters of $\mathrm{CD} 68^{+}$ macrophages and by multinucleated osteoclasts. We also investigated the OSM level in the plasma of NHO patients and demonstrated that the OSM median level in patient plasma was increased 2-fold compared with HD plasma (Figure 7B).

Since macrophage-derived OSM has been shown to stimulate osteogenic differentiation $(39,41$, 42), we analyzed the production of OSM by LPS-activated CD14 $4^{+}$monocytes/macrophages from

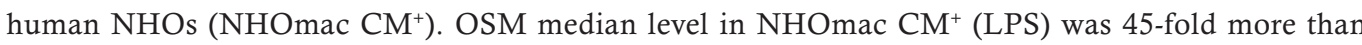
in NHOmac $\mathrm{CM}^{-}$(CT; Figure $\left.7 \mathrm{C}\right)$, suggesting that OSM secretion by NHO-associated $\mathrm{CD}_{14}^{+}$monocytes/macrophages is strongly stimulated by LPS. We then analyzed the expression of OSMRs on NHO-MDSCs and found that OSMR and CD130 (gp130) subunits were both expressed at the surface of these cells, confirming that they can respond to OSM (Figure 7D). We next showed that recombinant human OSM (10-100 $\mathrm{ng} / \mathrm{ml})$ increased mineralization of NHO-MDSCs compared with the osteogenic differentiation medium alone (Figure 7E). The stimulating effect of OSM on the osteoblastic differentiation of NHO-MDSCs was further confirmed by Western blot analysis showing that OSM increased expression of Runx2 as well as of osteocalcin, a marker of mature osteoblasts (Figure $7 F)$. We further tested whether OSM secreted by monocytes/macrophages from NHOs participated in the mineralization of NHO-MDSCs. For this purpose, NHOmac $\mathrm{CM}^{+}$was added (1:10 dilution) to the osteogenic differentiation medium and NHO-MDSCs in the presence of neutralizing mouse anti-human OSM monoclonal antibody $(10 \mu \mathrm{g} / \mathrm{ml})$ or nonimmune isotype-matched mouse IgG2a for 12 days. Addition of anti-OSM antibodies decreased 3-fold NHO-MDSC mineralization induced by the osteogenic differentiation medium supplemented with conditioned medium from LPS-activated monocytes/macrophages (Figure 7G).

These results suggest that OSM is involved in human NHO formation. 
A
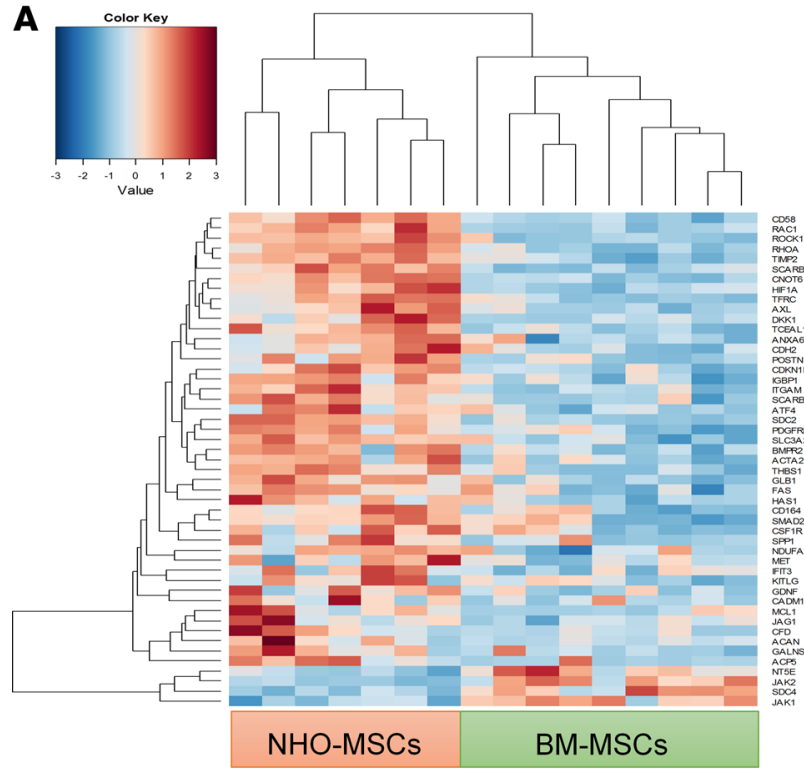

B

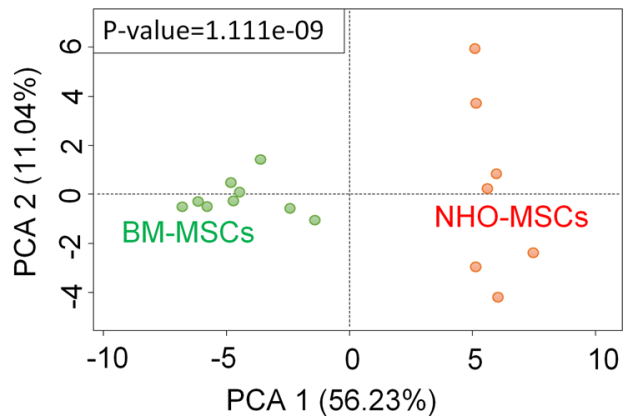

C
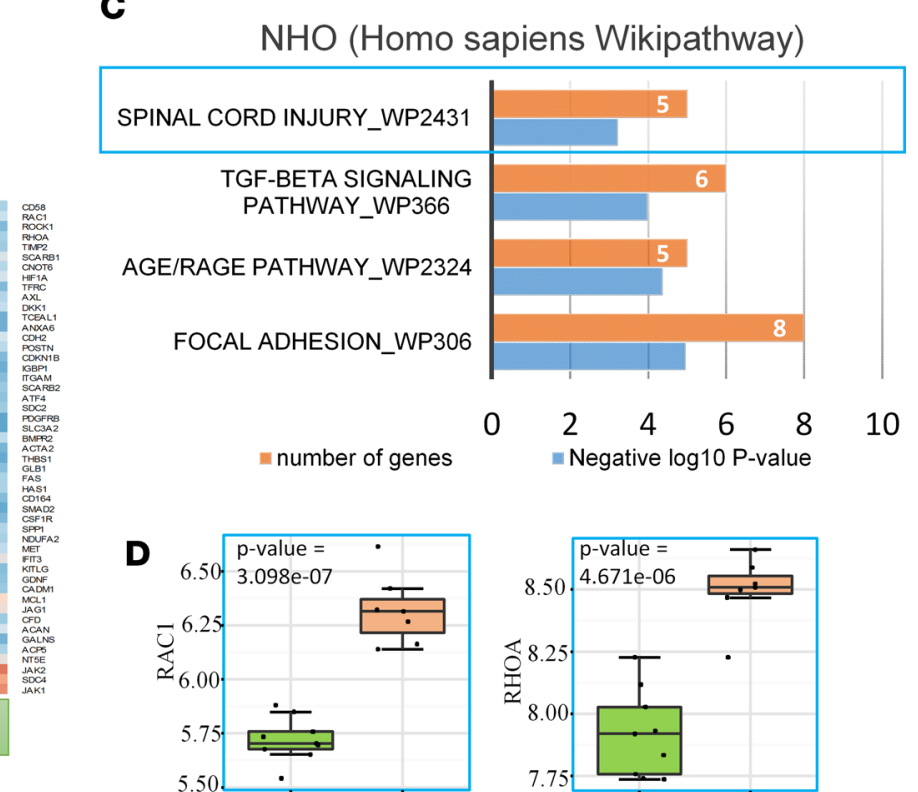

BM-MSCs NHO-MSCs
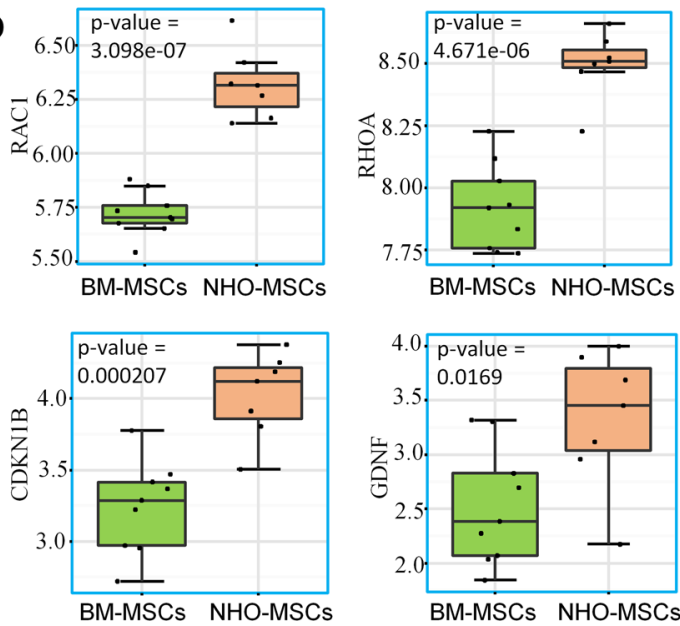

Figure 4. Spinal cord injury imprinting of human NHO-MSCs. (A) Transcriptome heatmap performed with bone marrow niche functionality-related genes differentially expressed between neurogenic heterotopic ossification (NHO) and bone marrow (BM) mesenchymal stromal cells (MSCs); unsupervised classification was performed with Euclidean distances. Dual-color scale from blue to red is correlated with mRNA level expression. (B) Principal component analysis performed with bone marrow niche-related genes differentially expressed between NHO-MSCs and BM-MSCs ( $P$ value of group discrimination was calculated with Pearson correlation of the group variable to the first principal component). (C) WikiPathway functional enrichment of BM niche-related genes upregulated in NHO-MSCs as compared with BM-MSCs ( $P=0.0006, P$ value were estimated by Fisher exact test with Enrichr application). (D) Box plot of genes modulated after spinal cord injury and found upregulated in NHO-MSCs as compared with BM-MSCs ( $P$ value was calculated with 2-tailed Student's $t$ test). The box-and-whisker plot shows median, 25th and 75th percentile, minimum and maximum values.

OSM accumulates at sites of NHO in mice. Finally, to confirm the role of OSM in NHO development, we used our mouse model of NHO, in which mice undergo spinal cord transection between T12-T14, together with muscle damage induced by intramuscular injection of cardiotoxin (CDTX) (11). In this model, NHOs develop in the injured muscle exclusively in mice with SCI and CDTX-induced muscle damage (SCI+CDTX) via macrophages infiltrating the injured muscle (11). In wild-type animals at day 4 after injury, Osm mRNA was significantly increased in the injured muscles of SCI+CDTX mice, whereas sham-operated mice with an intramuscular injection of CDTX (Sham+CDTX) had lower induction of Osm mRNA (Figure 8A, $P<0.01$ ). IHC staining of muscles from Sham+CDTX mice (Figure 8B) confirmed the absence of $\mathrm{NHO}$ at 21 days after surgery, with very little $\mathrm{F} 4 / 80^{+}$macrophage infiltration and little expression of collagen type 1 (Coll1), OSM, or osterix, suggesting that muscle is mostly repaired without NHO; likewise, similar results were seen in mice that underwent SCI without CDTX injection (Supplemental Figure 4). In sharp contrast, in SCI+CDTX mice, CDTX-injured hamstring muscle contained Coll1 ${ }^{+}$bone matrix and osterix ${ }^{+}$osteoblasts (Figure 8B). As previously shown (11), in SCI+CDTX mice, $\mathrm{F} 4 / 80^{+}$macrophages were intercalated among the Col11 ${ }^{+}$heterotopic bone foci (Figure $8 \mathrm{~B}$ ), and IHC for OSM confirmed increased expression around areas of NHO, particularly in areas with accumulation of $\mathrm{F} 4 / 80^{+}$macrophages (Figure $8 \mathrm{~B}$, circled area) and osterix ${ }^{+}$osteoblasts (Figure 8B, arrows). Overall, this suggests that OSM expression is increased in injured muscles of mice early after SCI, while 
A
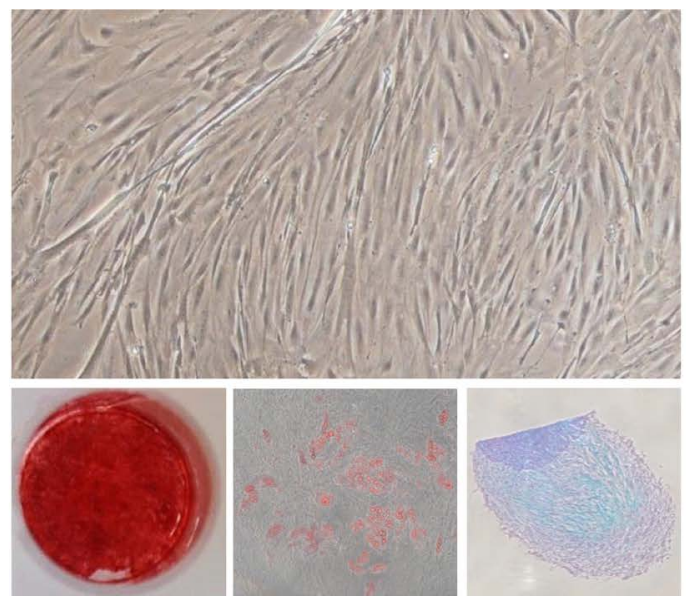

Alizarin Red S

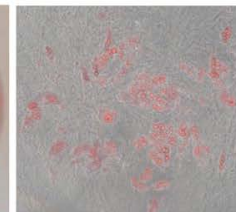

Oil Red

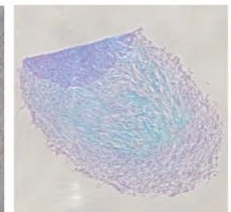

Alcian Blue
C

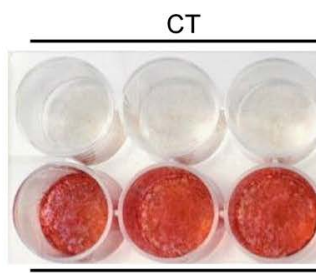

OB + LPS

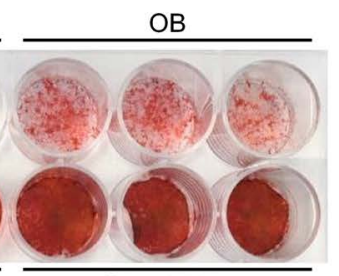

$\mathrm{OB}+\mathrm{TNFa}$

D

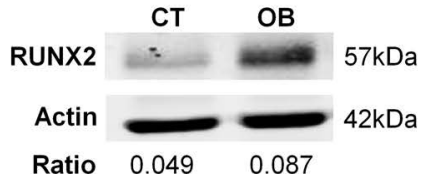

B
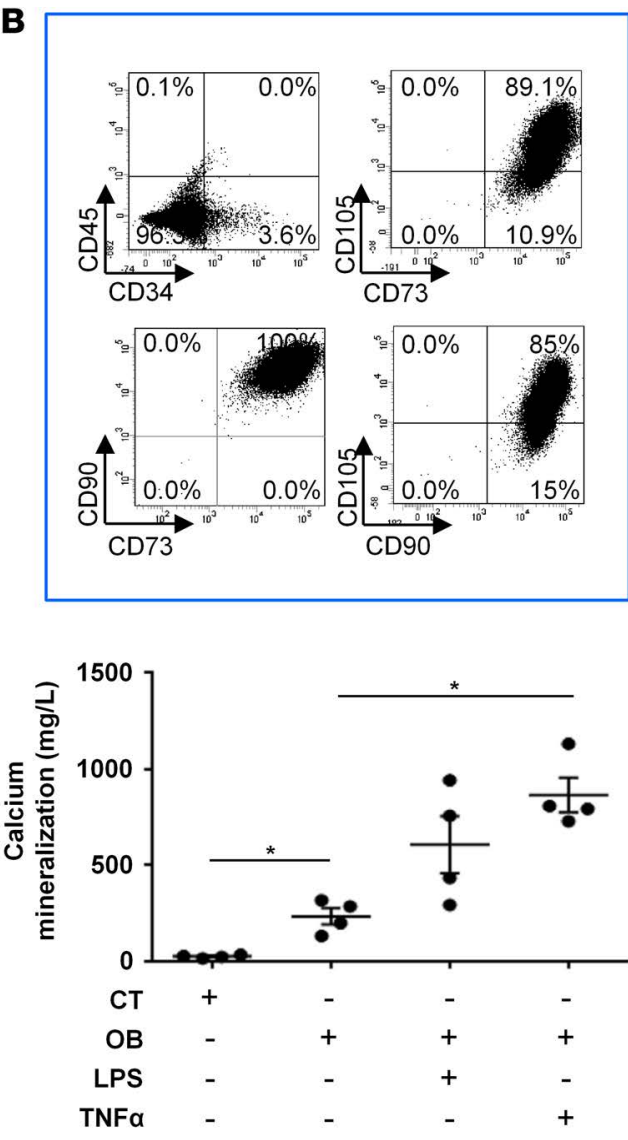

CT

OB

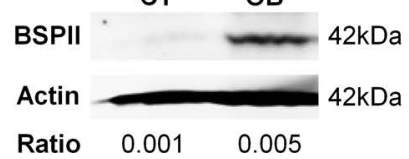

Figure 5. Osteogenic potential of human NHO-MDSCs in response to proinflammatory stimuli. (A) Neurogenic heterotopic ossification muscle-derived stromal cells (NHO-MDSCs) were isolated, cultured, and then subsequently induced to differentiate into 3 mesenchymal lineages using specific media. Differentiation into osteoblasts, adipocytes, and chondrocytes was evaluated by Alizarin Red S, Oil Red O, and Alcian blue staining, respectively. Original magnification, $\times 10$. (B) NHO-MDSCs express classical mesenchymal markers, as shown by flow cytometry. (C) NHO-MDSCs were cultured in control medium (CT) or osteogenic medium alone (OB) or were supplemented with LPS (100 ng/ml) (OB + LPS) or TNF- $\alpha(100 \mathrm{ng} / \mathrm{ml})(\mathrm{OB}+\mathrm{TNF}-\alpha)$ for $3 \mathrm{weeks}$. Cells were then stained with Alizarin Red S. Calcium mineralization was quantified and expressed as mean \pm SEM $(n=4)$. For statistical analysis, 1 -way ANOVA followed by Dunnett's post-hoc test were used $\left({ }^{*} P \leq 0.05\right.$, between experimental conditions). (D) Runx2 and BSPII protein expression by Western blot of NHO-MDSC cell lysates with (OB) or without (control [CT]) osteoblastic differentiation medium (day 3 and day 21, respectively). Ratios correspond to RUNX2/actin or BSPII/actin.

OSM protein is secreted and accumulates in the sites of HO development after SCI.

Deletion of the Osmr gene reduces NHO formation in response to SCI and muscular injury. To test the function of OSM signaling in vivo, mice genetically deficient for the OSMR gene (Osmr ${ }^{--}$mice) and their wild-type controls underwent spinal cord transection and CDTX injection as previously described (11). In Osmr ${ }^{-1}$ mice, NHO volume was significantly reduced compared with wild-type controls 7-14 days after injury (median $3.2 \mathrm{~mm}^{3}$ in $O s m r^{--}$versus $14.4 \mathrm{~mm}^{3}$ in wild-type, $P=0.0038$ ) (Figure 9, A and B), confirming that signaling through the OSMR promotes $\mathrm{HO}$ formation in this model.

Finally, we sorted CD45-Ter119-CD31-CD $34^{+}$Sca1 ${ }^{-}$SCs and CD $45^{-}$Ter119-CD $31^{-} \mathrm{CD} 34^{+} \mathrm{Sca} 1^{+}$interstitial cells (ICs) from the hamstring muscles of naive wild-type C57BL/6 mice and cultured them in osteogenic conditions as previously described (11), in the presence or absence of mouse recombinant OSM. Mouse OSM significantly increased the mineralizing potential of mouse muscle ICs and SCs. This suggests that, as in human muscle cells, OSM can promote mineralization and osteogenic differentiation of mouse muscle progenitor cells (Figure 9C). 
A

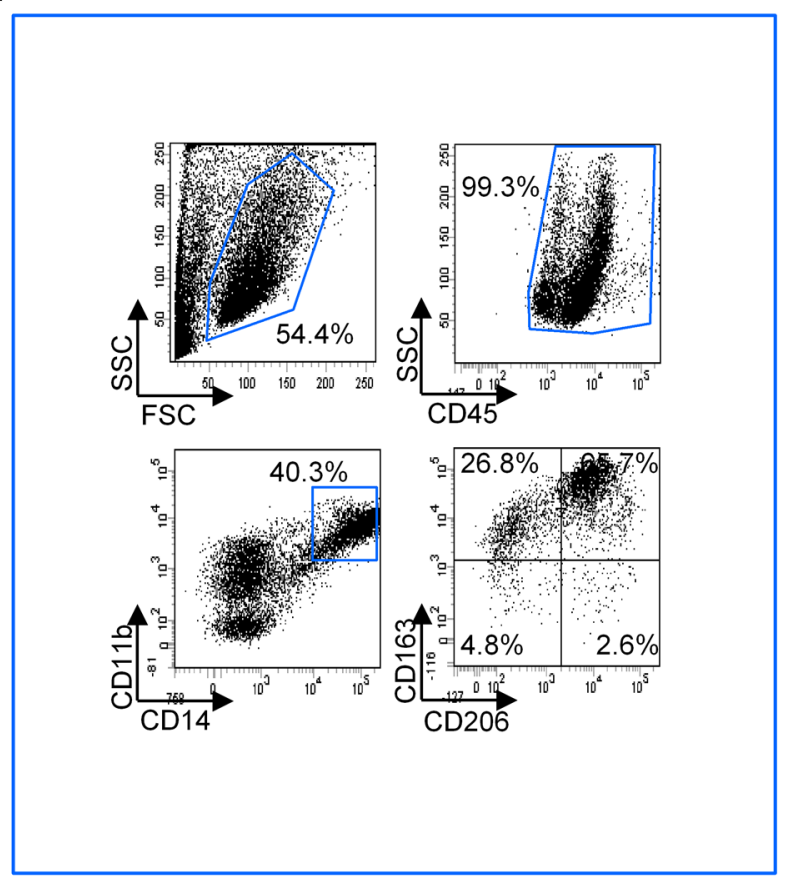

C

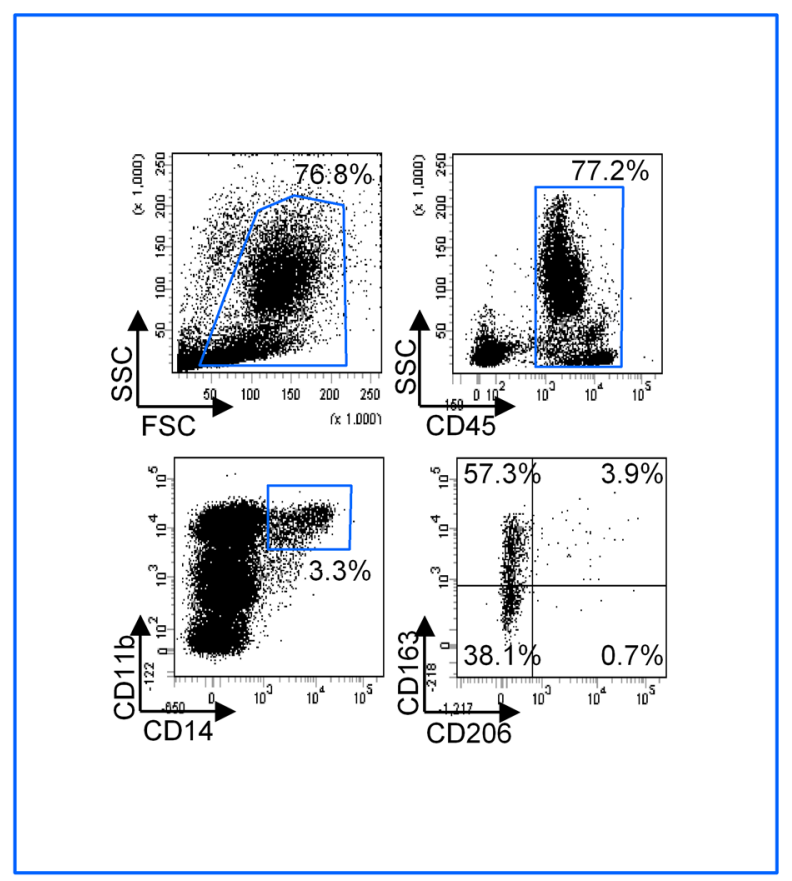

B
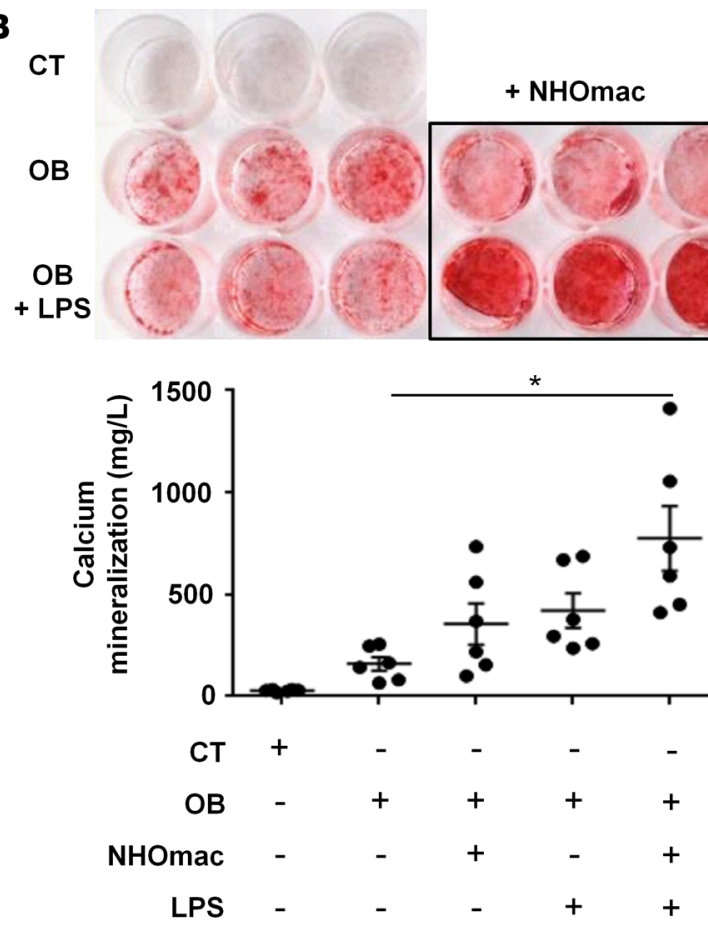

D CT OB
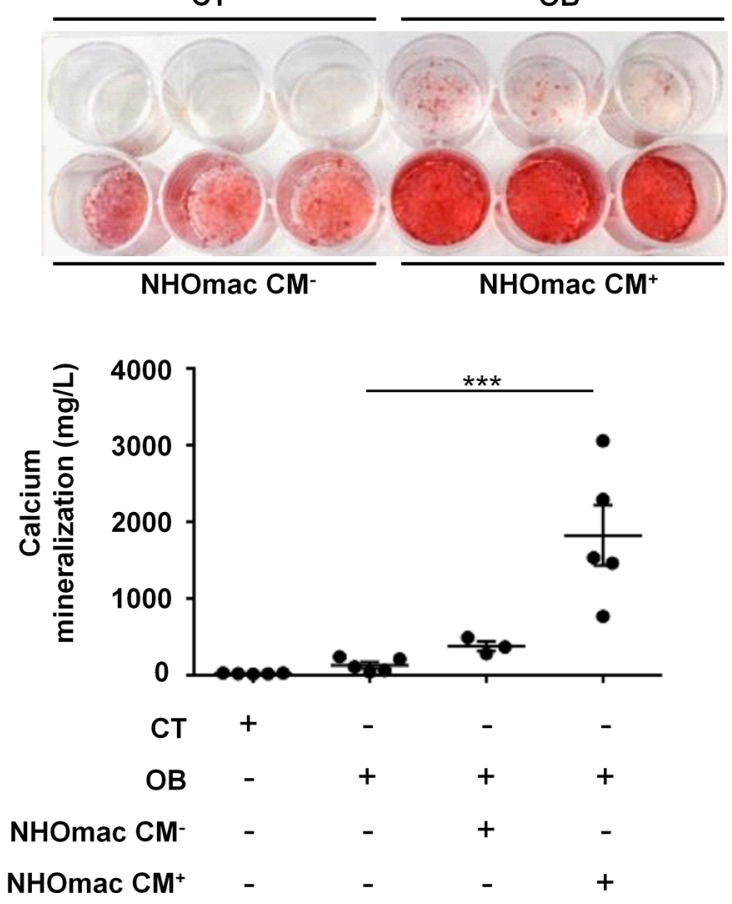

Figure 6. Activated macrophages contribute to the osteogenic differentiation of human NHO-MDSCs. (A) CD34 ${ }^{+}$cells from human neurogenic heterotopic ossification ( $\mathrm{NHO}$ ) were isolated using immunomagnetic cell separation and induced to differentiate into macrophages for 2 weeks in specific medium. Cells were then analyzed by flow cytometry for expression of myeloid marker CD11b, monocyte/macrophage marker CD14, and M2 macrophage activation marker CD163 and CD206. (B) Activated macrophages derived in vitro from NHO CD34 ${ }^{+}$cells stimulate NHO muscle-derived stromal cells (NHO-MDSCs). NHO-MDSCs were cultured in control medium (CT) or osteogenic medium alone (OB) or were supplemented with LPS (100

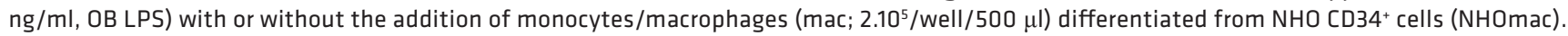
After 3 weeks, cells were then stained with Alizarin Red S. Calcium mineralization was quantified and expressed as mean \pm SEM ( $n=6$ ). (C) Mononuclear cells from human NHO marrows were analyzed by flow cytometry for mac markers, as in A. (D) Medium conditioned by activated macrophages from human NHO stimulate NHO-MDSC mineralization. In that purpose, CD14+ monocytes/macrophages from human NHO were isolated using immunomagnetic cell separation and cultured with or without LPS $(100 \mathrm{ng} / \mathrm{ml})$ for 3 days. Conditioned media with (NHOmac CM+) or without LPS (NHOmac $\mathrm{CM}^{-}$) were recovered and added to cultures of NHO-MDSCs. NHO-MDSCs were cultured in control medium (CT) or osteogenic medium alone 
(OB) or supplemented with NHOmac $\mathrm{CM}^{-}$or $\mathrm{NHOmac}^{\mathrm{CM}^{+}}$(diluted at 1:10) for 3 weeks. Cells were then stained with Alizarin Red S. Calcium mineralization was quantified and expressed as mean \pm SEM $(n \geq 3)$. For statistical analysis, 1-way ANOVA followed by Dunnett's post-hoc test were used ( ${ }^{*} P \leq 0.05$ and $\left.{ }^{* *} P \leq 0.001\right)$.

Altogether, our results strongly suggest that OSM is produced by activated macrophages in injured muscles in the context of SCI and promotes NHO development in humans and mice following SCI or traumatic brain injuries.

\section{Discussion}

We report herein that NHOs from patients contain hematopoietic niches in which a mesenchymal and endothelial environment supports HSC maintenance, proliferation, and differentiation. More importantly, our results from patients and mice genetically deficient for OSMR suggest that activated macrophages contribute to $\mathrm{NHO}$ formation through the osteogenic action of OSM on muscle cells within an inflammatory context.

The "cell of origin" of HO within the muscle is not well defined. In muscles from HDs, a subpopulation of $\mathrm{CD} 90^{-} \mathrm{MSCs}$ is reportedly capable of differentiating into osteoblasts, adipocytes, and chondrocytes under appropriate conditions in contrast to $\mathrm{CD} 90^{+} \mathrm{MSCs}$, which have limited adipogenic and chondrogenic differentiation potential (21). PDGFR $\alpha^{+}$cells, which exhibited an in vitro and in vivo osteogenic differentiation potential in normal muscles, have been observed on histological sections proximal to the HO-muscle interface in one trauma patient (18). In our study, patient-derived NHO-MDSCs expressed the classical CD73, CD105, and CD90 mesenchymal markers, and, similar to BM-MSCs, they could differentiate toward osteogenic and adipogenic lineages. NHO-MDSCs also had the capacity to produce cartilage extracellular matrix in vitro. Altogether, our results suggest that NHO-MDSCs are capable of participating in the formation of HOs in brain injury or SCI patients, as reported with MSCs isolated from battlefield-injured muscle tissue (19). NHOs can relapse, even 15 years after surgery (F. Genet, unpublished observations) (53), and our results indicate that NHO-MDSCs with osteogenic potential are still present in muscles after NHO resection. This hypothesis is strengthened by the recent study by Agarwal et al. (54), showing that PDGFR $\alpha^{+}$MSCs are responsible for $\mathrm{HO}$ recurrence in a burn/tenotomy mouse model. Thus, when reactivated under specific conditions, residual NHO-MDSCs may participate in NHO recurrence.

The inflammatory context that frequently accompanies neurogenic trauma (55) has been suggested to be a key actor of NHO development. Inflammatory and osteogenic cytokines are reported to be overexpressed in injured muscles following severe war injuries (56). Likewise, in our clinically relevant model of NHO in mice with SCI, macrophages in injured and inflamed muscles are key drivers of NHO development (11). Furthermore LPS, an inducer of the inflammatory response, slightly increased the mineralizing ability and osteoblastic differentiation of human NHO-MDSCs. These results are consistent with a previous report indicating that LPS weakly stimulated osteoblastic differentiation of BM-MSCs through LIF secretion (39). However, LPS-stimulated macrophages caused a marked increase in NHO-MDSC mineralization and osteoblastic differentiation, suggesting that macrophage activation is involved in this process (Figure 5). Our results using HO biopsies from brain injury or SCI patients confirm our previous study in mice showing the role of macrophage-mediated inflammation in our experimental model of SCI-induced $\mathrm{NHO}$ (11). Moreover, addition of medium conditioned by LPS-activated macrophages isolated from NHO $\left(\mathrm{NHOmac} \mathrm{CM}^{+}\right)$to NHO-MDSC culture medium elicited similar outcomes, indicating that activated macrophages in NHOs release osteogenic factors.

Among these osteogenic factors, OSM - a cytokine produced by several cell types, including activated monocytes/macrophages from peripheral blood and BM (39, 41, 42), osteoblasts, and osteocytes (38) - induces osteogenic differentiation of various cell types, including MSCs from BM or adipose tissues (38-40). OSM is synergistic with TNF- $\alpha$ on vascular calcification in vitro (57) and is required for normal bone formation in vivo, as shown in mice defective for the Osm gene (58) or for its receptor type II Osmr (38). In the current study, we show that OSM concentration is higher in the plasma of NHO patients and that macrophages isolated from patient NHO overproduce OSM after LPS activation in vitro, suggesting that both local and systemic levels of OSM are elevated in NHO. The role of OSM in osteogenic mineralization of NHO-MDSCs was confirmed by the use of neutralizing anti-OSM antibodies, suggesting that OSM produced locally by activated macrophages participates in the development of HOs from muscle cells in SCI or brain-injured patients. This is consistent with our observation that OSM is produced by clusters of $\mathrm{CD} 68^{+}$monocytes/macrophages in NHO histological sections. OSM expression was also 
A

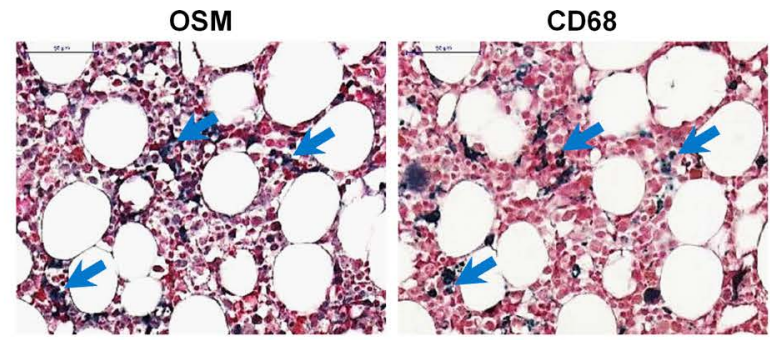

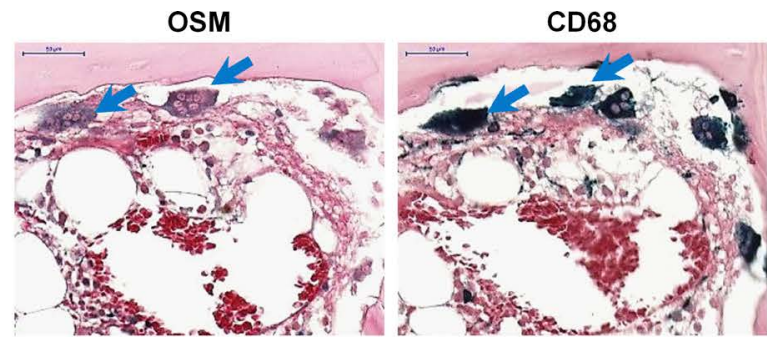
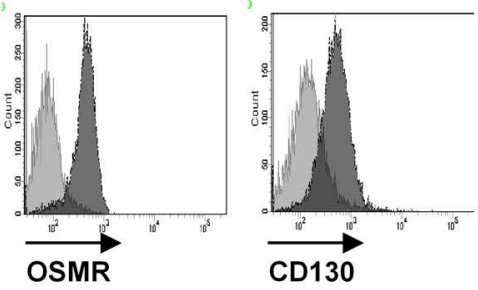

B

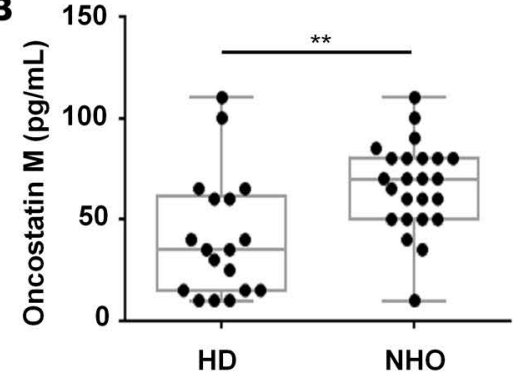

C

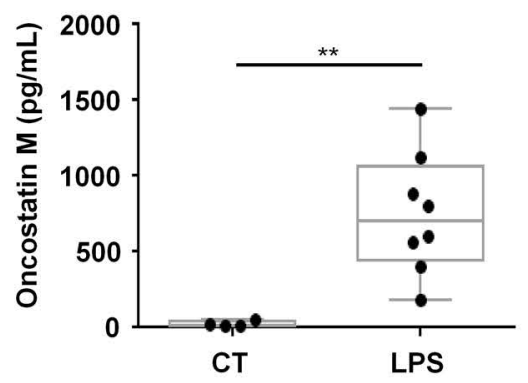

D

G

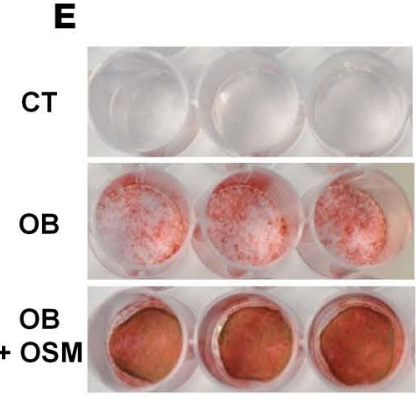

$\mathbf{F}$

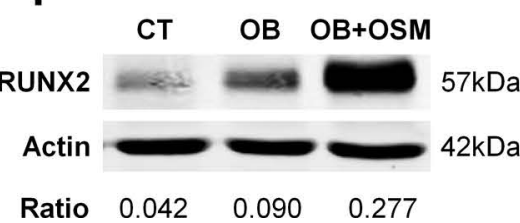

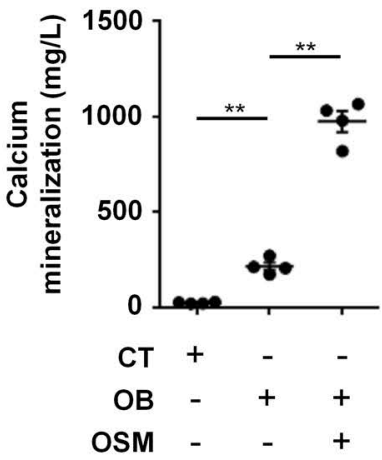

CT
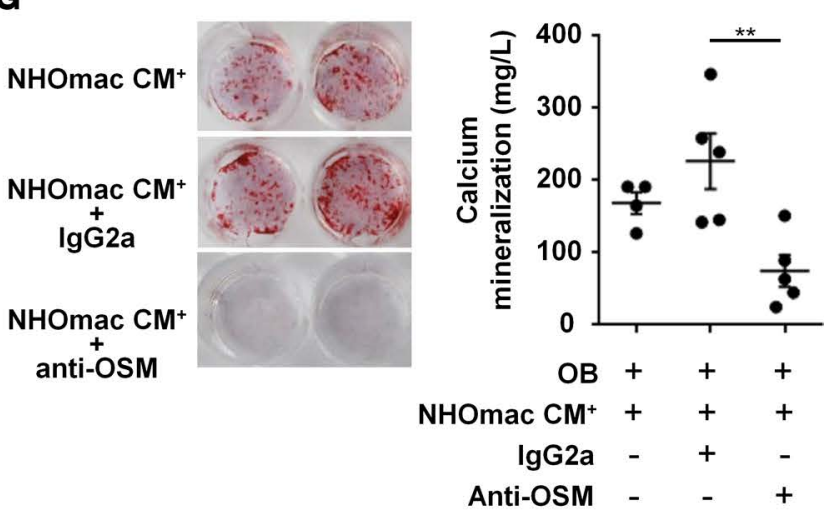

Figure 7. Macrophage-derived OSM is involved in human NHO formation. (A) CD68 and oncostatin M (OSM) staining on neurogenic heterotopic ossification (NHO) serial sections. Blue arrows indicate OSM or CD68 dark blue staining. Scale bar: $50 \mu \mathrm{m}$. (B) OSM concentrations measured by ELISA in blood plasma from healthy donors (HD) or NHO patients. Each dot represents a different donor/patient, and the box-and-whisker plot shows median, 25th and 75th percentile, minimum and maximum values $\left(n=18-24 ;{ }^{*} P \leq 0.01\right.$, nonparametric Mann-Whitney test). (C) NHO CD14 ${ }^{+}$monocytes/macrophages cultured with or without LPS ( $100 \mathrm{ng} / \mathrm{ml})$ for 3 days. OSM concentrations were measured in control (CT) or LPS-stimulated conditioned medium (LPS). Each dot is from a different donor ( $n=4-8$; ${ }^{* *} P \leq 0.01$, nonparametric Mann-Whitney test). (D) Expression of OSM receptor (OSMR) and CD130 (gp130) on NHO muscle-derived stromal cells (NHO-MDSCs) by flow cytometry. Light gray curves represent isotype-matched control antibodies. (E) NHO-MDSCs were cultured in control medium (CT) or osteogenic medium alone (OB) or supplemented with OSM (100 ng/ml, OB + OSM). Cells were then stained with Alizarin Red S. Calcium mineralization was quantified and expressed as mean \pm SEM $(n=4)$. (F) Western blots show Runx2 and osteocalcin (OC) protein expression in NHO-MDSC lysates stimulated or not with OSM and with (OB) or without (control [CT]) osteoblastic differentiation medium (day 3 and day 21, respectively). (C) Human NHO CD14 ${ }^{+}$monocytes/macrophages (mac) were cultured with LPS $(100 \mathrm{ng} / \mathrm{ml}$ ) for 3 days and conditioned media (NHOmac CM+) were recovered. NHO-MDSCs were cultured in osteogenic medium supplemented with $\mathrm{NHOmac} \mathrm{CM}^{+}$alone $\left(\mathrm{NHOmac}^{\mathrm{CM}} \mathrm{M}^{+}\right)$or in the presence of control $\mathrm{lgC}_{2 \mathrm{a}}\left(\mathrm{NHOmac} \mathrm{CM}^{+}+\mathrm{IgC}_{2 \mathrm{a}}\right)$ or anti-OSM $\left(\mathrm{NHOmac} \mathrm{CM}^{+}+\right.$anti-OSM) antibody for 12 days. Cells were then stained with Alizarin Red S. Calcium mineralization was expressed as mean \pm SEM $(n=4-5)$. Ratios correspond to RUNX2/actin or OC/actin. For statistical analysis, 1-way ANOVA followed by Dunnett's post-hoc test were used (E and $\mathbf{G})\left({ }^{* *} P \leq 0.01\right)$.

noted in osteoblasts and osteocytes in NHO samples (Supplemental Figure 5) and is also likely to further increase the local OSM concentration. This observation in mature human NHO is consistent with the increased expression of Osm mRNA in the injured muscle of mice 4 days after SCI as well as abundant OSM protein associated with both osterix ${ }^{+} \mathrm{NHO}$ osteoblasts and $\mathrm{F} 4 / 80^{+}$macrophages associated with 


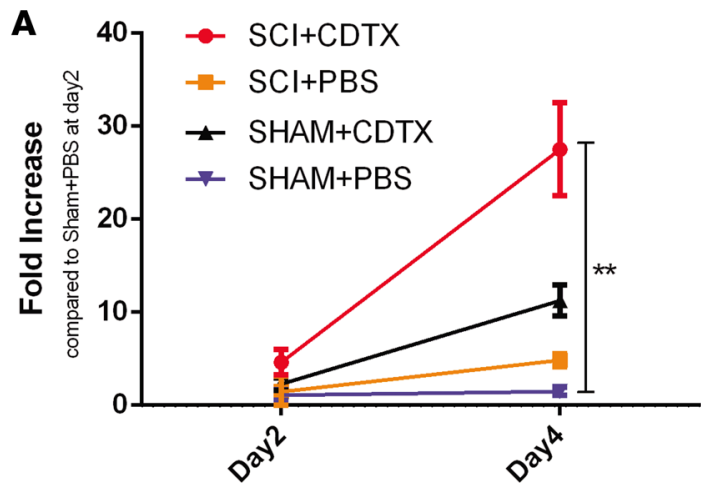

\section{Days after $\mathrm{SCl}$}

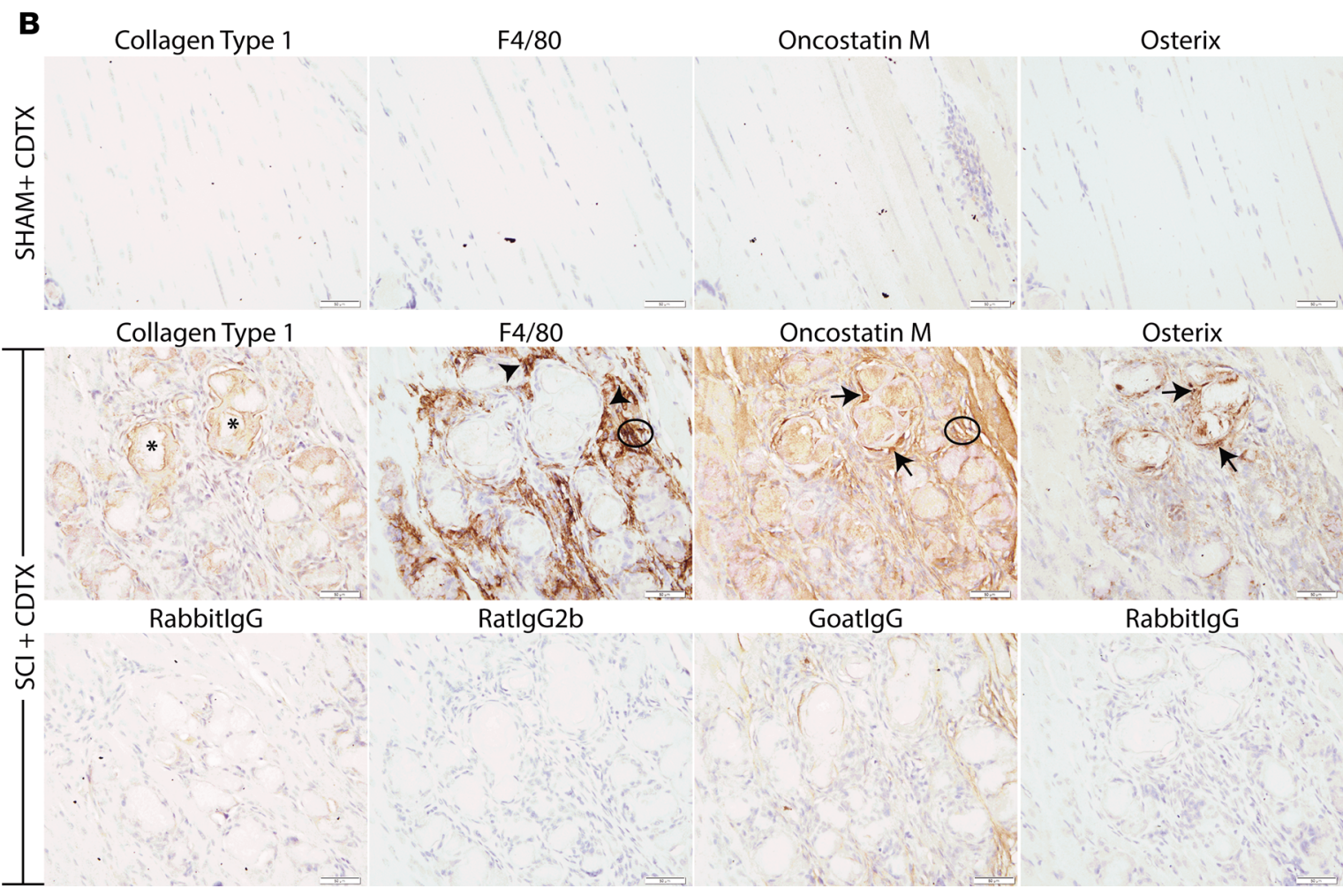

Figure 8. Oncostatin $\mathbf{M}$ is expressed at sites of $\mathbf{N H O}$ following $\mathbf{S C l}$ in mice. (A) Oncostatin $\mathrm{M}(\mathrm{Osm})$ mRNA expression is significantly increased in injured muscle after spinal cord injury (SCI). Total RNA was extracted from right hamstring muscle from mice at days 2 and 4 after $\mathrm{SCl}$ or sham surgery and intramuscular cardiotoxin (CDTX) or PBS injection ( $n=3 / 4$ mice/group, 1 experiment). Results show qRT-PCR quantification of Osm mRNA (relative to $\beta$-actin) with a significant increase in Osm mRNA in muscle 4 days after SCI+CDTX compared with SHAM+PBS mice (**P<0.01 Kruskal-Wallis test). (B) Representative IHC images of hind limbs from mice that underwent sham or SCI surgery with an intramuscular injection of CDTX. At 21 days after surgery in SHAM+CDTX mice, no neurogenic heterotopic ossification (NHO) is noted in the hamstrings, with few macrophages and absence of osterix or OSM expression. In $\mathrm{SCl}+\mathrm{CDTX}$ mice, collagen type $1^{+}$(Coll1 $1^{+}$) bone foci were noted within the muscle (asterisks); surrounding this $\mathrm{NHO}$ are numerous $\mathrm{F} 4 / 80^{+}$macrophages (arrowheads). IHC for OSM confirmed expression of OSM around areas of NHO, with OSM expression noted in areas of macrophage accumulation (circled area) and osterix ${ }^{+}$osteoblasts (arrows). Specificity of staining was confirmed with matched isotype controls (first RabbitlgG: Coll1, RatlgC2b:F4/80, GoatlgG:OSM, and second RabbitlgG:Osterix). Data in A are represented as mean \pm SD. Original magnification: $\times 40$. Scale bar: $50 \mu \mathrm{m}$.

NHO 21 days after SCI. Of note, OSM protein staining was diffuse in the injured muscle of SCI mice developing $\mathrm{HO}$, consistent with the observation that bioactive OSM binds to extracellular matrix proteins, such as collagen I and laminin (59), which are abundant in muscle, particularly at acidic $\mathrm{pH}$, as found in inflamed or injured tissues. Of note, deletion of the Osmr gene did not completely ablate NHO, as observed following in vivo macrophage depletion with clodronate liposomes (11). This suggests that other cytokines or mechanisms involving macrophages are also at play to promote NHO development in addition to OSM. Among them, the participation of BMP2 in a potential crosstalk with OSM remains to 
A

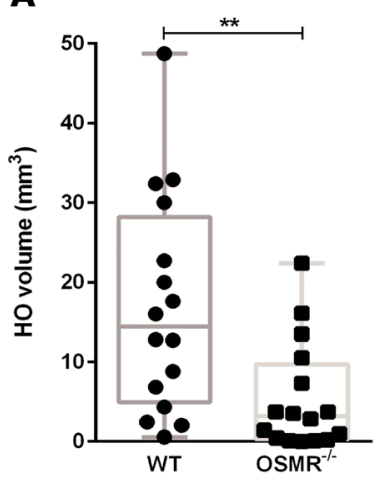

B

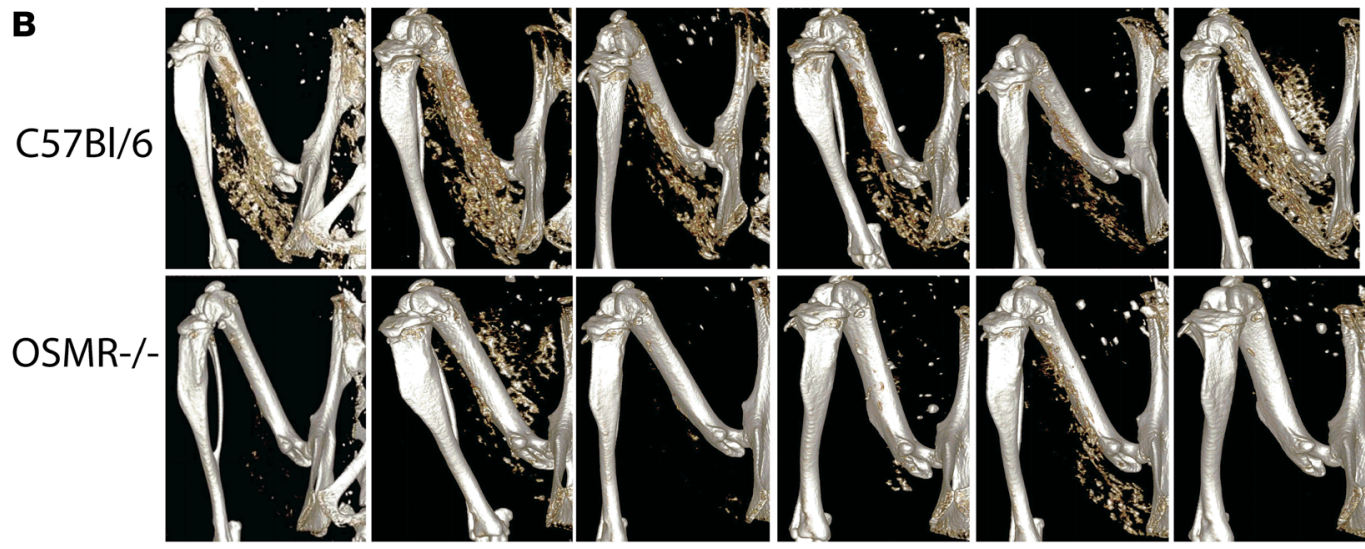

C
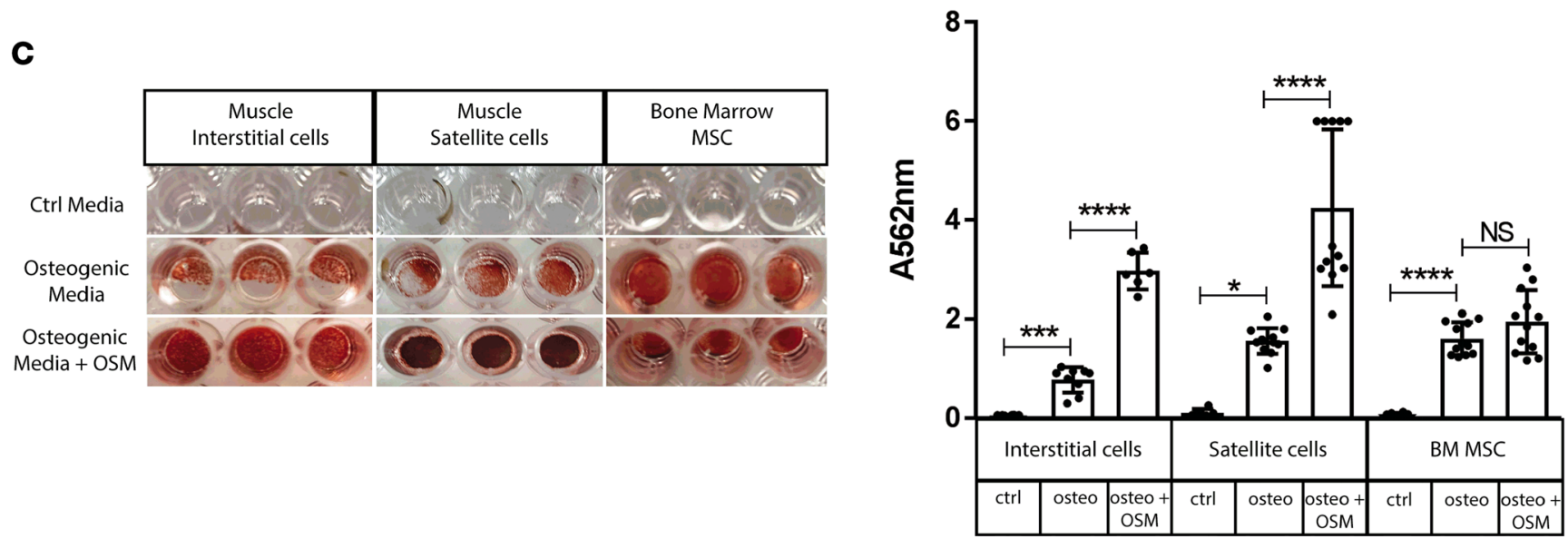

Figure 9. Deletion of the $\mathbf{O s m r}$ gene reduces NHO following SCI in mice. (A) $\mu \mathrm{CT}$ analysis of neurogenic heterotopic ossification (NHO) development 7-14 days following spinal cord injury (SCI) and intramuscular cardiotoxin (CDTX) injection in oncostatin M receptor null (Osmr ${ }^{-1-}$ ) mice compared with wild-type C57BL/6 control mice. Each dot represents an individual mouse, and the box-and-whisker plot shows median, 25 th and 75 percentile, and minimum and maximum values. Statistical significance was confirmed using a Mann-Whitney test $(P=0.0038,16$ mice/group, experiment repeated twice). (B) Representative 3D reconstructed images of $\mathrm{NHO}$ in $\mathrm{C57BL} / 6$ and $0 \mathrm{smr} \mathrm{r}^{-/}$mice 7 days after surgery. (C) Recombinant mouse oncostatin $\mathrm{M}$ (OSM) enhances in vitro osteogenic differentiation. Muscle interstitial cells, satellite cells, and bone marrow mesenchymal stromal cells (BM-MSCs) sorted from naive C57BL/6 mice were cultured for 10 days in control medium, osteogenic medium, or osteogenic medium plus mouse $0 S \mathrm{M}$ (25 $\mathrm{ng} / \mathrm{ml}$ ). Quantification of Alizarin Red S staining via absorbance at $562 \mathrm{~nm}$ confirmed enhanced calcium deposition in interstitial cells ( $\left.{ }^{* * *} P<0.01\right)$, satellite cells $\left({ }^{*} P<0.05\right)$, and BM-MSCs $\left({ }^{* * *} P<0.001\right)$ in the presence of osteogenic media alone and significantly enhanced calcium deposition in both interstitial cells and satellite cells in the presence of osteogenic media plus OSM (**** $P<0.001)$. Results are the mean and SDs of 2 separate experiments ( $n=3$ control media and $n=3-6$ osteogenic media \pm OSM/each experiment).

be explored (60). Nevertheless, the fact that $\mathrm{NHO}$ volume is reduced 3-fold in $\mathrm{Osmr}^{-1-}$ mice demonstrates that OSM/OSMR pathway is an important promoter of NHO development.

By playing multiple critical roles on hematopoietic microenvironment, OSM is reported to regulate hematopoiesis in the BM $(43,44,61)$. In the present study, we show that human NHOs represent a suitable microenvironment for maintaining quiescent HSCs and determining their activation state and fate, therefore meeting the definition of HSC niches. Indeed, we show that CD $34^{+} \mathrm{HSCs}$, with a quiescent SP phenotype $(45,48,62)$ and the ability to reconstitute human hematopoiesis in vivo when injected in immunodeficient mice, dwell in human NHOs. It is likely that these NHO-resident HSCs come from the circulation and home to NHO using a suitable mesenchymal and endothelial microenvironment (niches) similar to that existing in the BM. MSCs and endothelial cells are essential components of medullar HSC niches (63-66) and play a major role in HSC maintenance by secreting key cytokines, such as CXCL12 (67). In that respect, it is interesting to note that CXCL12 levels are increased in NHO patient plasma (Supplemental Figure 6) and that CXCL12's expression has been reported to be stimulated by OSM in MSCs (68). Beside their capacity for AOC differentiation, human NHO-MSCs exhibit similar molecular and functional characteristics to BM-MSCs in terms of long-term human hematopoiesis support and SP functionality induction in vitro (48). More importantly, when seeded on hydroxyapatite scaffolds and 
subcutaneously implanted into immunodeficient mice (69), human NHO-MSCs reconstituted HSC niches supportive of murine hematopoietic cells confirming their role in supporting hematopoiesis in vivo. Our results definitively demonstrate the presence of functional HSCs within human NHOs.

In conclusion, the present study demonstrates that OSM, a cytokine required to maintain the microenvironment for hematopoiesis, is secreted by activated macrophages in NHO. It participates in the osteogenic differentiation of human muscle cells from NHO patients in an inflammatory context and in the formation of NHO in a murine model. This suggests that macrophages play a key role in the NHO development observed in brain injury or SCI patients and that OSM or OSMR could be a suitable therapeutic target.

Moreover, we demonstrate that human NHOs contain marrow tissue in which HSCs are maintained by a functional stromal microenvironment, thus documenting that NHOs are ectopic HSC niches. The evidence of hematopoietic cells and niches in ectopic bones growing at the expense of soft tissue in brain-injured or SCI patients strongly argues in favor of the brain-bone-blood triad concept proposed by Lapidot (70) and provides two dimensions to this triad, through muscles and inflammation.

\section{Methods}

\section{Human samples}

NHOs and muscles surrounding NHOs from 64 patients with brain injuries or SCI, strokes, or cerebral anoxia were sampled at Garches Hospital, Garches, France (Supplemental Table 4). BM cells and unmobilized peripheral blood samples from HDs were obtained at Blois Hospital (Blois, France) after total hip surgery and at Centre de Transfusion Sanguine des Armées (Percy Hospital, Clamart, France), respectively.

\section{Reagents}

Human recombinant OSM and TNF- $\alpha$, LPS and neutralizing anti-human OSM monoclonal antibody (clone 17001), and recombinant mouse OSM were purchased from Miltenyi Biotec, Invivogen, and R\&D Systems, respectively.

Immunomagnetic cell separations

NHOs were cut into small pieces, and cells were extracted in RPMI1640 medium (Thermo Fisher Scientific). Mononuclear cells from NHO, BM, or unmobilized peripheral blood were recovered after Pancoll gradient $\left(1.077 \mathrm{~g} / \mathrm{ml}\right.$; Dutscher). Immunomagnetic $\mathrm{CD} 34^{+}, \mathrm{CD} 31^{+}$, or $\mathrm{CD} 14^{+}$cell separations were performed according to the manufacturer's instructions (Miltenyi Biotec).

\section{Isolation of NHO-MSCs and NHO-MDSCs}

NHO explants were seeded in $\alpha$-MEM (Clinisciences) supplemented with $10 \% \mathrm{FCS}$ and $1 \%$ antibiotics (50 $\mu \mathrm{g} / \mathrm{ml}$ penicillin, $50 \mu \mathrm{g} / \mathrm{ml}$ streptomycin, and $100 \mu \mathrm{g} / \mathrm{ml}$ neomycin) at $37^{\circ} \mathrm{C}$ in $5 \% \mathrm{CO}_{2}$. Sprouting adherent cells were trypsinized at subconfluence and seeded at 4,000 per $\mathrm{cm}^{2}$.

Adherent cells from muscles surrounding NHOs, named NHO-MDSCs, were trypsinized at subconfluence and seeded at 4,000 per $\mathrm{cm}^{2}$.

\section{MSC transcriptomic analysis and bioinformatics}

Total RNA from NHO-MSCs (7 samples) and BM-MSCs (9 samples) was extracted by the Nucleospin RNA II protocol from Macharey Nagel. Transcriptomic analysis and bioinformatics were performed as described in the Supplemental Methods.

\section{Tissue collection for histology and IHC analysis}

For human NHOs. Biopsies were fixed in 4\% formaldehyde, demineralized in hydrochloric and formic acid, and embedded in paraffin. 4- $\mu \mathrm{m}$ sections were cut on the Cochin HistIM Facility, and IHC was carried out as described in the Supplemental Methods.

For mouse tissues. At 7-21 days after surgery, mice were euthanized by $\mathrm{CO}_{2}$ asphyxiation and the right hind limb was fixed in freshly made $4 \%$ paraformaldehyde (Sigma-Aldrich) at $4^{\circ} \mathrm{C}$ for 48 hours and transferred to PBS for micro-computed tomography $(\mu \mathrm{CT})$ imaging. For histologic analysis, bones were 
decalcified for at least 2 weeks in 14\% EDTA (Sigma-Aldrich), pH 7.2. Once decalcified, all specimens were processed for paraffin embedding. IHC was carried out as previously described $(11,38,71)$ and as described in the Supplemental Methods.

\section{Flow cytometry}

Cells were incubated in a FACS buffer composed of PBS, 2\% FCS, and 2 mM EDTA on ice for 20 minutes with specific antibodies (Supplemental Table 5). Flow cytometry analysis was carried out on BD Fortessa apparatus using FACSDiva software (BD Biosciences).

\section{SP cell detection}

For SP cell detection in human NHOs, mononuclear cells were isolated on a Pancoll gradient. Lineage-negative ( $\mathrm{Lin}^{-}$) cells were recovered using the lineage depletion kit (Miltenyi Biotec) according to the manufacturer's instructions. SP population cell detection was performed as described in the Supplemental Methods.

\section{Hematopoietic colony formation and long-term culture-initiating cell assays}

CD $34^{+}$cells $\left(10^{3} / \mathrm{ml}\right)$ isolated from human NHOs, nonmobilized peripheral blood, or BM were seeded in methylcellulose medium with cytokines according to the manufacturer's instructions (MethoCult H4034 Optimum, Stemcell Technologies). CFU were scored after 14 days at $37^{\circ} \mathrm{C}$ in $5 \% \mathrm{CO}_{2}$ atmosphere using an inverted microscope.

Long-term culture-initiating cell assays were performed as described in the Supplemental Methods.

Xenografts of $\mathrm{CD}^{+} 4^{+}$cells from human NHOs transplanted into immunodeficient mice Nonobese diabetic severe combined immunodeficient $\mathrm{Il2rg}^{-/-}$(NOD.CB17-Prkdc ${ }^{\text {scid }}$ Il2 $\mathrm{rg}^{\mathrm{tm} 1 \mathrm{Wj} 1}$ or NSG) mice (Janvier Labs) were conditioned and grafted as described in the Supplemental Methods.

\section{Isolation of human endothelial cells}

$\mathrm{CD} 1^{+}$cells from human NHOs were recovered after immunomagnetic CD31 cell separation and were stained with specific antibodies (Supplemental Table 5). Endothelial CD $31^{+} \mathrm{CD} 144^{+} \mathrm{CD} 34^{+} \mathrm{CD} 45^{-}$cells were then sorted using FACSAria III (BD Biosciences) and incubated in EGM-2 MV medium (EGM2MV SingleQuots, Lonza) for 1 month. Matrigel assays as well as ICAM-1 and VCAM-1 expression were performed on human endothelial cells as described in the Supplemental Methods.

\section{In vivo MSC osteogenic assays}

Subconfluent human NHO-MSCs were collected for subcutaneous implantation into the flanks of 10 -week-old female nude mice (Janvier Labs). Implants (at least $n=4$ per group) were prepared by mixing sterile $80-200 \mu \mathrm{m}$ particles (60\% hydroxyapatite/40\% $\beta$-tricalcium phosphate) (Graftys), $100 \mu \mathrm{l}$ human plasma, and $10^{6}$ human NHO-MSCs as described previously (69). Ten microliters of $2 \% \mathrm{CaCl}_{2}$ $2 \mathrm{H}_{2} \mathrm{O}$ was added to obtain coagulation. Ten weeks after implantation, animals were sacrificed and the scaffolds were collected. Scaffolds were decalcified and H\&E staining was done for histologic evaluation.

\section{In vitro osteogenic differentiation and quantification of mineralized calcium}

Human stromal cells were seeded in 24-well plates at 3,000 per $\mathrm{cm}^{2}$ in $\alpha$-MEM supplemented with $10 \%$ FCS and $1 \%$ antibiotics. After cell adhesion, medium was removed and replaced by $\alpha$-MEM supplemented with $10 \% \mathrm{FCS}, 1 \%$ antibiotics and $0.052 \mu \mathrm{g} / \mathrm{ml}$ dexamethasone, $12.8 \mu \mathrm{g} / \mathrm{ml}$ ascorbic acid, and $2.15 \mathrm{mg} /$ $\mathrm{ml} \beta$-glycerophosphate from Sigma-Aldrich. Cells were cultured for 12 days to 3 weeks at $37^{\circ} \mathrm{C}$ in $5 \% \mathrm{CO}_{2}$ atmosphere, and medium was changed 2 times in a week. Quantification of mineralization was performed after Alizarin Red S staining as described in the Supplemental Methods.

\section{In vitro adipogenic and chondrogenic differentiation}

Human stromal cells were seeded either at 21,000 per $\mathrm{cm}^{2}$ or at $2.5 \times 10^{5}$ in a conical tube (volume 15 $\mathrm{ml}$ ) for in vitro adipogenic or chondrogenic differentiation, respectively, in $\alpha$-MEM supplemented with $10 \%$ FCS and $1 \%$ antibiotics, as described in the Supplemental Methods for in vitro adipogenic and chondrogenic differentiation, respectively. 


\section{CD34+ cell differentiation into macrophages}

$\mathrm{CD}^{+}{ }^{+}$cells isolated from human NHOs were seeded at $10^{5}$ per $\mathrm{ml}$ in 24 -well plates and cultured in SynH medium (ABCell-Bio) supplemented with SCF, thrombopoietin, Flt3L, and IL-3 (10 ng/ml each, Miltenyi Biotec) for 1 week at $37^{\circ} \mathrm{C}$ in $5 \% \mathrm{CO}_{2}$ atmosphere. Cells were centrifuged and resuspended in IMDM supplemented with $20 \%$ FCS, $1 \%$ antibiotics, $25 \mathrm{ng} / \mathrm{ml} \mathrm{SCF}, 30 \mathrm{ng} / \mathrm{ml} \mathrm{M-CSF}, 30 \mathrm{ng} / \mathrm{ml} \mathrm{Flt3L}$, and $30 \mathrm{ng} /$ $\mathrm{ml}$ IL-3 (all from Miltenyi Biotec). Cells were seeded at $10^{5}$ per $\mathrm{ml}$ in 24-well plates and cultured for an additional week at $37^{\circ} \mathrm{C}$ in $5 \% \mathrm{CO}_{2}$ atmosphere.

\section{Preparation of monocyte/macrophage-conditioned media}

$\mathrm{CD} 14^{+}$cells isolated from human NHOs were seeded at $1.25 \times 10^{6}$ per $\mathrm{ml}$ in 24 -well plates and cultured in $\alpha$-MEM supplemented with $10 \%$ FCS and $1 \%$ antibiotics and in the presence or absence of LPS (100 ng/ $\mathrm{ml}$ ) at $37^{\circ} \mathrm{C}$ in $5 \% \mathrm{CO}_{2}$ atmosphere as previously described (39). After 3 days, supernatants were centrifuged to remove cells and frozen at $-80^{\circ} \mathrm{C}$. Supernatants of $\mathrm{CD} 14^{+}$cells stimulated with LPS were named $\mathrm{NHOmac} \mathrm{CM}^{+}$and supernatants of $\mathrm{CD}_{14}{ }^{+}$cells without stimulation were named $\mathrm{NHOmac} \mathrm{CM}^{-}$.

\section{OSM level evaluation by ELISA}

OSM levels were evaluated in blood samples from HDs and from patients with NHOs as well as in conditioned media of $\mathrm{CD}_{14}{ }^{+}$macrophages isolated from NHOs by ELISA as described in the Supplemental Methods.

\section{Western blot}

Cells were lysed in RIPA (Abcam) or Laemmli buffer. Proteins (20 or $30 \mu \mathrm{g})$ were loaded on a SDS-polyacrylamide gel and transferred to a PVDF membrane. Primary antibodies included an anti-RUNX2 (rabbit monoclonal antibody, clone D1H7, Cell Signaling), anti-BSPII (rabbit polyclonal antibody, 5468, Cell Signaling), anti-osteocalcin (mouse monoclonal antibody, clone OC4-30, Abcam), or anti- $\beta$-actin (mouse monoclonal antibody, clone 6D1, MBL). Alexa Fluor 680 anti-rabbit or anti-mouse (A21076 and A21058, Thermo Fisher Scientific) or DyLight 800 anti-mouse (\#35521, Thermo Fisher Scientific) antibodies were added to detect specific proteins using an Odyssey scanner (LI-COR Biosciences).

\section{$\mathrm{NHO}$ mouse model}

C57BL/ 6 mice and Osmr ${ }^{-1-}$ mice (backcrossed over 10 times in C57BL/6 background) (6-9 weeks old) were used in all experiments. C57BL/ 6 mice were obtained from the Animal Resource Center (Perth, Australia), and $\mathrm{Osmr}^{-1}$ mice were provided by NAS. Mice were anesthetized by intraperitoneal injection of $100 \mathrm{mg} / \mathrm{kg}$ ketamine and $10 \mathrm{mg} / \mathrm{kg}$ xylazine in sterile saline. Alaminectomy was performed on the dorsal spine and the spinal cord was transected with a scalpel blade between T11 and T13. Control animals were anesthetized and sham operated with an incision in the dorsal skin and muscle. Immediately after surgery, while still anesthetized, mice were then injected with CDTX purified from Naja pallida venom (Latoxan) at $0.1568-0.3125 \mathrm{mg} / \mathrm{kg}$ or PBS in the right hamstring. Mice received a subcutaneous injection of ciprofloxacin $(10 \mathrm{mg} / \mathrm{kg})$ and recovered on a heating pad. As SCI causes paraplegia, mouse bladders were expressed manually by gentle massage of the bladder twice daily throughout the experiments. Mice were given Bactrim ( $800 \mathrm{mg} / 1$, Roche) in drinking water following surgical procedures as prophylaxis for bladder infections.

\section{Mouse $\mu \mathrm{CT}$}

NHO volume was measured in vivo or ex vivo using the Inveon PET-CT multimodality system (Siemens Medical Solutions Inc.). The parameters used were as follow: $360^{\circ}$ rotation, 180 projections, $80 \mathrm{kV}$ voltage, $500 \mu \mathrm{A}$ current, and effective pixel size $36 \mu \mathrm{m}$. 3D images were reconstructed by the $\mu \mathrm{CT}$ system software package. For in vivo imaging, mice were anesthetized by $2 \%$ isoflurane during the imaging process. $\mathrm{HO}$ volume was analyzed in the Inveon Research Workplace (Siemens Medical Solutions Inc.).

Mouse muscle cell cultures, osteogenic differentiation, and quantification CD45-Ter119-CD31-CD34+Sca1- SCs and CD45-Ter119-CD31-CD34+ Sca $1^{+}$ICs from mouse muscles and mesenchymal cells from BM of naive C57BL/6 mice were isolated and sorted by flow cytometry as previously described (11). SCs and ICs were cultured in high glucose DMEM (GIBCO Invitrogen 11960-044) 
containing 20\% (v/v) FBS, 10\% horse serum, and $1 \mathrm{X}$ penicillin-streptomycin-glutamine (PSG; at $1 \mathrm{U}$ penicillin/ml, $1 \mu \mathrm{g} / \mathrm{ml}$ streptomycin, and $0.2 \mathrm{mM}$ glutamine) (GIBCO Invitrogen, 10378). BM-MSCs were cultured in $\alpha$-MEM containing $20 \%$ (v/v) FBS (GIBCO Invitrogen, 10099-141) and 1X PSG. All 3 cell types were then cultured under osteogenic conditions ( $\alpha$-MEM [10\% (v/v) FBS, $10 \mathrm{mM} \beta$-glycerophosphate, $200 \mu \mathrm{M}$ phosphoascorbic acid, $2 \mathrm{mM} \mathrm{CaCl}_{2}$, and $0.2 \mu \mathrm{M}$ dexamethasone]) for 14 days, in the presence or absence of recombinant mouse OSM at $25 \mathrm{ng} / \mathrm{ml}$ (R\&D Systems). Calcium deposition was quantified using Alizarin Red S as described in the Supplemental Methods.

\section{Mouse mRNA extraction and qRT-PCR analysis}

Muscle samples (right hamstring muscles) were homogenized in Trizol (Invitrogen), and mRNAs for Osm and Actb ( $\beta$-actin) were measured as described in the Supplemental Methods.

\section{Statistics}

For comparisons of two groups, significance was calculated by the nonparametric Mann-Whitney test or by 2-tailed Student's $t$ test. For comparisons of more than two groups, significance was calculated either by 1-way or 2-way ANOVA or Kruskal-Wallis test using PRISM 6 software (GraphPad software). $P \leq 0.05$ was considered significant.

\section{Study approval}

For human studies, all samples were obtained with the informed consent of patients and according to good clinical practices with approval from the people protection committee (CPP 09025) and from the national commission for informatics and Liberties (CNIL Eyo1066211J). For animal studies, the use and care of animals was approved by the French Institutional Animal Care and Use Committee or by the Animal Experimentation Ethics Committee of the University of Queensland.

\section{Author contributions}

FT, JPL, JJL, and MCLBK designed the study; FT and BG performed experiments on human samples and analyzed results; FT, AA, and LB performed grafts of human $\mathrm{CD}_{3} 4^{+}$cells in mice and analyzed results; AA performed SP cell assays, and NA performed endothelial cell culture and identification; SS performed Western blot experiments; SB provided advice for muscle cell isolation and culture; C Desterke designed and performed MSC transcriptomic, molecular biology, and bioinformatics analyses as well as online big data submission; DC performed endothelial cell sorting and analyzed results; NR, AA, and MCLBK performed scaffold graft experiments in mice, and FT, NR, AA and MCLBK analyzed results; EV, GG, C Debaud, PD, FG, and JJL provided human samples; KAA, HWT, IK, MS, and BJ performed experiments in the mouse model of SCI-induced NHO; KAA also performed osteogenic assays and IHC in the mouse model; ARP performed IHC staining for mouse collagen I, F4/80, and osterix; NAS provided Osmr/- mice and advice on the experiments in mice; and FT, JPL, JJL, and MCLBK wrote and edited the manuscript. All authors approved the manuscript.

\section{Acknowledgments}

This research was funded by project grant 1101620 from the French Government Defense Procurement and Technology Agency (DGA; to FT, FG, JJL, and MCLBK), project grant APP1101620 from the National Health and Medical Research Council of Australia (NHMRC to JPL, FG, and NAS), and by the Assistant Secretary of Defense for Health Affairs through Spinal Cord Injury Research Program under award no. W81XWH-15-1-0606 from the US Department of Defence; UMR-S-MD1197 is partly funded by INSERM. JPL is supported by research fellowship APP1044091 from the NHMRC. The Translational Research Institute is partly funded by the Federal Government of Australia. The authors are indebted to P. Mauduit (Inserm UMR-S-MD1197, Paul Brousse Hospital, Villejuif, France) for his scientific and technical advices concerning Western blot analyses. The authors greatly acknowledge the technical assistance of "Cochin HistIM Facility" (Paris, France) and D. Burgot (Polyclinique de Blois, La Chaussée-Saint-Victor, France) for supplying human BM samples. The authors also acknowledge the scientific and technical assistance of the Translational Research Institute Preclinical Imaging Facility, which is supported by Therapeutic Innovation Australia (TIA). TIA is supported by the Australian Government through the National Collaborative Research Infrastructure Strategy program. 
Address correspondence to: Marie-Caroline Le Bousse-Kerdilès, Inserm UMR-S-MD1197, Hôpital Paul Brousse, Bâtiment Lavoisier, 14 avenue Paul-Vaillant Couturier, 94807 Villejuif Cedex, France. Phone: 33.1.4559.5303; Email: caroline.le-bousse-kerdiles@inserm.fr. Or to: Jean-Pierre Levesque, University of Queensland, Mater Research Institute, Translational Research Institute, Woolloongabba, Queensland 4102, Australia. Phone: 61.7.3443.7571; Email: jp.levesque@mater.uq.edu.au.

1. Shore EM, et al. A recurrent mutation in the BMP type I receptor ACVR1 causes inherited and sporadic fibrodysplasia ossificans progressiva. Nat Genet. 2006;38(5):525-527.

2. Fukuda T, et al. Constitutively activated ALK2 and increased SMAD1/5 cooperatively induce bone morphogenetic protein signaling in fibrodysplasia ossificans progressiva. J Biol Chem. 2009;284(11):7149-7156.

3. van Dinther M, et al. ALK2 R206H mutation linked to fibrodysplasia ossificans progressiva confers constitutive activity to the BMP type I receptor and sensitizes mesenchymal cells to BMP-induced osteoblast differentiation and bone formation. $J$ Bone Miner Res. 2010;25(6):1208-1215.

4. Chakkalakal SA, et al. An Acvr1 R206H knock-in mouse has fibrodysplasia ossificans progressiva. J Bone Miner Res. 2012;27(8):1746-1756.

5. Pek CH, Lim MC, Yong R, Wong HP. Neurogenic heterotopic ossification after a stroke: diagnostic and radiological challenges. Singapore Med J. 2014;55(8):e119-e122.

6. Genêt F, Chehensse C, Jourdan C, Lautridou C, Denormandie P, Schnitzler A. Impact of the operative delay and the degree of neurologic sequelae on recurrence of excised heterotopic ossification in patients with traumatic brain injury. J Head Trauma Rehabil. 2012;27(6):443-448.

7. Brady RD, Shultz SR, McDonald SJ, O’Brien TJ. Neurological heterotopic ossification: Current understanding and future directions [published online ahead of print May 16, 2017]. Bone. https://doi.org/10.1016/j.bone.2017.05.015.

8. Wang D, Shurafa MS, Acharya R, Strand VF, Linden MD. Chronic abdominal pain caused by heterotopic ossification with functioning bone marrow: a case report and review of the literature. Arch Pathol Lab Med. 2004;128(3):321-323.

9. Christofi T, Raptis DA, Kallis A, Ambasakoor F. True trilineage haematopoiesis in excised heterotopic ossification from a laparotomy scar: report of a case and literature review. Ann R Coll Surg Engl. 2008;90(5):W12-W14.

10. Davis TA, Lazdun Y, Potter BK, Forsberg JA. Ectopic bone formation in severely combat-injured orthopedic patients -- a hematopoietic niche. Bone. 2013;56(1):119-126.

11. Genêt F, et al. Neurological heterotopic ossification following spinal cord injury is triggered by macrophage-mediated inflammation in muscle. J Pathol. 2015;236(2):229-240.

12. Boulais PE, Frenette PS. Making sense of hematopoietic stem cell niches. Blood. 2015;125(17):2621-2629.

13. Chalmers J, Gray DH, Rush J. Observations on the induction of bone in soft tissues. J Bone Joint Surg Br. 1975;57(1):36-45.

14. Kan L, Kessler JA. Evaluation of the cellular origins of heterotopic ossification. Orthopedics. 2014;37(5):329-340.

15. Suda RK, et al. Circulating osteogenic precursor cells in heterotopic bone formation. Stem Cells. 2009;27(9):2209-2219.

16. Otsuru S, Tamai K, Yamazaki T, Yoshikawa H, Kaneda Y. Bone marrow-derived osteoblast progenitor cells in circulating blood contribute to ectopic bone formation in mice. Biochem Biophys Res Commun. 2007;354(2):453-458.

17. Wosczyna MN, Biswas AA, Cogswell CA, Goldhamer DJ. Multipotent progenitors resident in the skeletal muscle interstitium exhibit robust BMP-dependent osteogenic activity and mediate heterotopic ossification. J Bone Miner Res. 2012;27(5):1004-1017.

18. Oishi T, et al. Osteogenic differentiation capacity of human skeletal muscle-derived progenitor cells. PLoS ONE. 2013;8(2):e56641.

19. Nesti LJ, et al. Differentiation potential of multipotent progenitor cells derived from war-traumatized muscle tissue. J Bone Joint Surg Am. 2008;90(11):2390-2398.

20. Davis TA, O’Brien FP, Anam K, Grijalva S, Potter BK, Elster EA. Heterotopic ossification in complex orthopaedic combat wounds: quantification and characterization of osteogenic precursor cell activity in traumatized muscle. J Bone Joint Surg Am. 2011;93(12):1122-1131.

21. Downey J, et al. Prospective heterotopic ossification progenitors in adult human skeletal muscle. Bone. 2015;71:164-170

22. Bragdon B, Moseychuk O, Saldanha S, King D, Julian J, Nohe A. Bone morphogenetic proteins: a critical review. Cell Signal. 2011;23(4):609-620.

23. Leblanc E, et al. BMP-9-induced muscle heterotopic ossification requires changes to the skeletal muscle microenvironment. J Bone Miner Res. 2011;26(6):1166-1177.

24. Anthonissen J, Ossendorf C, Ritz U, Hofmann A, Rommens PM. Animal models for acquired heterotopic ossification. Acta Orthop Belg. 2014;80(1):2-10.

25. Kan L, Kessler JA. Animal models of typical heterotopic ossification. J Biomed Biotechnol. 2011;2011:309287.

26. Evans $\mathrm{KN}$, et al. Inflammatory cytokine and chemokine expression is associated with heterotopic ossification in high-energy penetrating war injuries. J Orthop Trauma. 2012;26(11):e204-e213.

27. Edwards DS, Clasper JC. Heterotopic ossification: a systematic review. J R Army Med Corps. 2015;161(4):315-321.

28. Slaets $\mathrm{H}$, et al. Oncostatin $\mathrm{M}$ reduces lesion size and promotes functional recovery and neurite outgrowth after spinal cord injury. Mol Neurobiol. 2014;50(3):1142-1151.

29. Guo S, et al. Oncostatin M confers neuroprotection against ischemic Stroke. J Neurosci. 2015;35(34):12047-12062.

30. Blanchard F, Duplomb L, Baud'huin M, Brounais B. The dual role of IL-6-type cytokines on bone remodeling and bone tumors. Cytokine Growth Factor Rev. 2009;20(1):19-28.

31. Gearing DP, et al. The IL-6 signal transducer, gp130: an oncostatin M receptor and affinity converter for the LIF receptor. Science. 1992;255(5050):1434-1437.

32. Mosley B, et al. Dual oncostatin M (OSM) receptors. Cloning and characterization of an alternative signaling subunit conferring 
OSM-specific receptor activation. J Biol Chem. 1996;271(51):32635-32643.

33. Richards CD. The enigmatic cytokine oncostatin $\mathrm{m}$ and roles in disease. ISRN Inflamm. 2013;2013:512103.

34. Suda T, et al. Oncostatin M production by human dendritic cells in response to bacterial products. Cytokine. 2002;17(6):335-340

35. Grenier A, et al. Oncostatin M production and regulation by human polymorphonuclear neutrophils. Blood. 1999;93(4):1413-1421.

36. Lisignoli G, et al. Osteoblasts and stromal cells isolated from femora in rheumatoid arthritis (RA) and osteoarthritis (OA) patients express IL-11, leukaemia inhibitory factor and oncostatin M. Clin Exp Immunol. 2000;119(2):346-353.

37. Repovic P, Benveniste EN. Prostaglandin E2 is a novel inducer of oncostatin-M expression in macrophages and microglia J Neurosci. 2002;22(13):5334-5343.

38. Walker EC, et al. Oncostatin M promotes bone formation independently of resorption when signaling through leukemia inhibitory factor receptor in mice. J Clin Invest. 2010;120(2):582-592.

39. Guihard P, et al. Induction of osteogenesis in mesenchymal stem cells by activated monocytes/macrophages depends on oncostatin M signaling. Stem Cells. 2012;30(4):762-772.

40. Song HY, Jeon ES, Kim JI, Jung JS, Kim JH. Oncostatin M promotes osteogenesis and suppresses adipogenic differentiation of human adipose tissue-derived mesenchymal stem cells. J Cell Biochem. 2007;101(5):1238-1251.

41. Nicolaidou V, et al. Monocytes induce STAT3 activation in human mesenchymal stem cells to promote osteoblast formation. PLoS ONE. 2012;7(7):e39871.

42. Fernandes TJ, et al. Cord blood-derived macrophage-lineage cells rapidly stimulate osteoblastic maturation in mesenchymal stem cells in a glycoprotein-130 dependent manner. PLoS ONE. 2013;8(9):e73266.

43. Tanaka M, Hirabayashi Y, Sekiguchi T, Inoue T, Katsuki M, Miyajima A. Targeted disruption of oncostatin M receptor results in altered hematopoiesis. Blood. 2003;102(9):3154-3162.

44. Sato F, Miyaoka Y, Miyajima A, Tanaka M. Oncostatin M maintains the hematopoietic microenvironment in the bone marrow by modulating adipogenesis and osteogenesis. PLoS One. 2014;9(12):e116209.

45. Goodell MA, Brose K, Paradis G, Conner AS, Mulligan RC. Isolation and functional properties of murine hematopoietic stem cells that are replicating in vivo. J Exp Med. 1996;183(4):1797-1806.

46. Charbord P, et al. A systems biology approach for defining the molecular framework of the hematopoietic stem cell niche. Cell Stem Cell. 2014;15(3):376-391.

47. Sutherland HJ, Eaves CJ, Lansdorp PM, Thacker JD, Hogge DE. Differential regulation of primitive human hematopoietic cells in long-term cultures maintained on genetically engineered murine stromal cells. Blood. 1991;78(3):666-672.

48. Malfuson JV, et al. SP/drug efflux functionality of hematopoietic progenitors is controlled by mesenchymal niche through VLA-4/CD44 axis. Leukemia. 2014;28(4):853-864.

49. Holzapfel BM, et al. Tissue engineered humanized bone supports human hematopoiesis in vivo. Biomaterials. 2015;61:103-114.

50. Youssefian T, et al. Nodular osteochondrogenic activity in soft tissue surrounding osteoma in neurogenic para osteo-arthropathy: morphological and immunohistochemical study. BMC Musculoskelet Disord. 2004;5:46.

51. Beauchamp JR, et al. Expression of CD34 and Myf5 defines the majority of quiescent adult skeletal muscle satellite cells. J Cell Biol. 2000;151(6):1221-1234.

52. Stec M, et al. Expansion and differentiation of CD14+CD16(-) and CD14+ +CD16+ human monocyte subsets from cord blood CD34+ hematopoietic progenitors. J Leukoc Biol. 2007;82(3):594-602.

53. Almangour W, Schnitzler A, Salga M, Debaud C, Denormandie P, Genêt F. Recurrence of heterotopic ossification after removal in patients with traumatic brain injury: A systematic review. Ann Phys Rehabil Med. 2016;59(4):263-269.

54. Agarwal S, et al. Surgical excision of heterotopic ossification leads to re-emergence of mesenchymal stem cell populations responsible for recurrence. Stem Cells Transl Med. 2017;6(3):799-806.

55. Mautes AE, Weinzierl MR, Donovan F, Noble LJ. Vascular events after spinal cord injury: contribution to secondary pathogenesis. Phys Ther. 2000;80(7):673-687.

56. Jackson WM, et al. Cytokine expression in muscle following traumatic injury. J Orthop Res. 2011;29(10):1613-1620.

57. Shioi A, et al. Induction of bone-type alkaline phosphatase in human vascular smooth muscle cells: roles of tumor necrosis factor-alpha and oncostatin M derived from macrophages. Circ Res. 2002;91(1):9-16.

58. Guihard P, et al. Oncostatin M, an inflammatory cytokine produced by macrophages, supports intramembranous bone healing in a mouse model of tibia injury. Am J Pathol. 2015;185(3):765-775.

59. Ryan RE, et al. Oncostatin M binds to extracellular matrix in a bioactive conformation: implications for inflammation and metastasis. Cytokine. 2015;72(1):71-85.

60. Zou F, et al. Effects of oncostatin M on cell proliferation and osteogenic differentiation in C3H10T1/2. J Musculoskelet Neuronal Interact. 2016;16(4):377-385.

61. Minehata $\mathrm{K}$, et al. Oncostatin m maintains the hematopoietic microenvironment and retains hematopoietic progenitors in the bone marrow. Int J Hematol. 2006;84(4):319-327.

62. Pierre-Louis O, et al. Dual SP/ALDH functionalities refine the human hematopoietic Lin-CD34+CD38-stem/progenitor cell compartment. Stem Cells. 2009;27(10):2552-2562.

63. Méndez-Ferrer S, et al. Mesenchymal and haematopoietic stem cells form a unique bone marrow niche. Nature. 2010;466(7308):829-834.

64. Ding L, Saunders TL, Enikolopov G, Morrison SJ. Endothelial and perivascular cells maintain haematopoietic stem cells. Nature. 2012;481(7382):457-462.

65. Ding L, Morrison SJ. Haematopoietic stem cells and early lymphoid progenitors occupy distinct bone marrow niches. Nature. 2013;495(7440):231-235.

66. Greenbaum A, et al. CXCL12 in early mesenchymal progenitors is required for haematopoietic stem-cell maintenance. Nature 2013;495(7440):227-230.

67. Ehninger A, Trumpp A. The bone marrow stem cell niche grows up: mesenchymal stem cells and macrophages move in. $J$ Exp Med. 2011;208(3):421-428.

68. Lee MJ, Song HY, Kim MR, Sung SM, Jung JS, Kim JH. Oncostatin M stimulates expression of stromal-derived factor-1 in 
human mesenchymal stem cells. Int J Biochem Cell Biol. 2007;39(3):650-659.

69. Bouvet-Gerbettaz S, et al. Adaptive immune response inhibits ectopic mature bone formation induced by $\mathrm{BMSCs} / \mathrm{BCP} / \mathrm{plasma}$ composite in immune-competent mice. Tissue Eng Part A. 2014;20(21-22):2950-2962.

70. Lapidot T, Kollet O. The brain-bone-blood triad: traffic lights for stem-cell homing and mobilization. Hematology Am Soc Hematol Educ Program. 2010;2010:1-6.

71. Chang MK, et al. Osteal tissue macrophages are intercalated throughout human and mouse bone lining tissues and regulate osteoblast function in vitro and in vivo. J Immunol. 2008;181(2):1232-1244. 